\title{
Asymptotic Fixed-Speed Reduced Dynamics for Kinetic Equations in Swarming
}

\author{
Mihai Bostan *, J. A. Carrillo ${ }^{\dagger}$
}

(August 25, 2018)

\begin{abstract}
We perform an asymptotic analysis of general particle systems arising in collective behavior in the limit of large self-propulsion and friction forces. These asymptotics impose a fixed speed in the limit, and thus a reduction of the dynamics to a sphere in the velocity variables. The limit models are obtained by averaging with respect to the fast dynamics. We can include all typical effects in the applications: short-range repulsion, long-range attraction, and alignment. For instance, we can rigorously show that the Cucker-Smale model is reduced to the Vicsek model without noise in this asymptotic limit. Finally, a formal expansion based on the reduced dynamics allows us to treat the case of diffusion. This technique follows closely the gyroaverage method used when studying the magnetic confinement of charged particles. The main new mathematical difficulty is to deal with measure solutions in this expansion procedure.
\end{abstract}

Keywords: Vlasov-like equations, Measure solutions, Swarming, Cucker-Smale model, Vicsek model, Laplace-Beltrami operator.

AMS classification: 92D50, 82C40, 92C10.

*Laboratoire d'Analyse, Topologie, Probabilités LATP, Centre de Mathématiques et Informatique CMI, UMR CNRS 7353, 39 rue Frédéric Joliot Curie, 13453 Marseille Cedex 13 France. E-mail : bostan@cmi.univ-mrs.fr

${ }^{\dagger}$ ICREA (Institució Catalana de Recerca i Estudis Avançats) and Departament de Matemàtiques, Universitat Autònoma de Barcelona, 08193 Bellaterra Spain. E-mail : carrillo@mat.uab.es. On leave from: Department of Mathematics, Imperial College London, London SW7 2AZ, UK. 


\section{Introduction}

This paper is devoted to continuum models for the dynamics of systems involving living organisms such as flocks of birds, school of fish, swarms of insects, myxobacteria... The individuals of these groups are able to organize in the absence of a leader, even when starting from disordered configurations [37]. Several minimal models describing such self-organizing phenomenon have been derived [38, 28, 19]. Most of these models include three basic effects: short-range repulsion, long-range attraction, and reorientation or alignment, in various ways, see [33] and particular applications to birds [32] and fish [1, 2].

We first focus on populations of individuals driven by self-propelling forces and pairwise attractive and repulsive interaction [34, 25]. We consider self-propelled particles with Rayleigh friction [17, 16, 11, 14, leading to the Vlasov equation in $d=2,3$ dimensions:

$$
\partial_{t} f^{\varepsilon}+v \cdot \nabla_{x} f^{\varepsilon}+a^{\varepsilon}(t, x) \cdot \nabla_{v} f^{\varepsilon}+\frac{1}{\varepsilon} \operatorname{div}_{v}\left\{f^{\varepsilon}\left(\alpha-\beta|v|^{2}\right) v\right\}=0, \quad(t, x, v) \in \mathbb{R}_{+} \times \mathbb{R}^{d} \times \mathbb{R}^{d}
$$

where $f^{\varepsilon}=f^{\varepsilon}(t, x, v) \geq 0$ represents the particle density in the phase space $(x, v) \in \mathbb{R}^{d} \times \mathbb{R}^{d}$ at any time $t \in \mathbb{R}_{+}, a^{\varepsilon}$ stands for the acceleration

$$
a^{\varepsilon}(t, \cdot)=-\nabla_{x} U \star \rho^{\varepsilon}(t, \cdot), \quad \rho^{\varepsilon}(t, \cdot)=\int_{\mathbb{R}^{d}} f^{\varepsilon}(t, \cdot, v) \mathrm{d} v,
$$

and $U$ is the pairwise interaction potential modelling the repelling and attractive effects. Here, the propulsion and friction forces coefficients $\alpha^{\varepsilon}=\frac{\alpha}{\varepsilon}>0, \beta^{\varepsilon}=\frac{\beta}{\varepsilon}>0$ are scaled in such a way that for $\varepsilon \rightarrow 0$ particles will tend to move with asymptotic speed $\sqrt{\frac{\alpha}{\beta}}$. These models have been shown to produce complicated dynamics and patterns such as mills, double mills, flocks and clumps, see [25]. Assuming that all individuals move with constant speed also leads to spatial aggregation, patterns, and collective motion [21, 26].

Another source of models arises from introducing alignment at the modelling stage. A popular choice in the last years to include this effect is the Cucker-Smale reorientation procedure [20]. Each individual in the group adjust their relative velocity by averaging with all the others. This velocity averaging is weighted in such a way that closer individuals in space have more influence than further ones. The continuum kinetic version of them leads to Vlasov-like models of the form (1) in which the acceleration is of the form

$$
a^{\varepsilon}(t, \cdot)=-H \star f^{\varepsilon}(t, \cdot),
$$

where $\star$ stands for the $(x, v)$-convolution, abusing a bit on the notation, with the nonnegative interaction kernel $H: \mathbb{R}^{2 d} \longrightarrow \mathbb{R}^{d}$. In the original Cucker-Smale work, the interaction is modelled by $H(x, v)=h(x) v$, with the weight function $h$ being a decreasing radial 
nonnegative function. We refer to the extensive literature in this model for further details [31, 29, 12, 13, 35].

In this work, we will consider the Vlasov equation (1) where the acceleration includes the three basic effects discussed above, and then takes the form:

$$
a^{\varepsilon}(t, \cdot)=-\nabla_{x} U \star \rho^{\varepsilon}(t, \cdot)-H \star f^{\varepsilon}(t, \cdot) .
$$

We will assume that the interaction potential $U \in C_{b}^{2}\left(\mathbb{R}^{d}\right), U$ bounded continuous with bounded continuous derivatives up to second order, and $H(x, v)=h(x) v$ with $h \in C_{b}^{1}\left(\mathbb{R}^{d}\right)$ and nonnegative. Under these assumptions the model (1)-(2) can be rigorously derived as a mean-field limit [36, 9, 24, 10, 3] from the particle systems introduced in [25, 20].

We will first study in detail the linear problem, assuming that the acceleration $a=a(t, x)$ is a given global-in-time bounded smooth field. We investigate the regime $\varepsilon \searrow 0$, that is the case when the propulsion and friction forces dominate the potential interaction between particles. At least formally we have

$$
f^{\varepsilon}=f+\varepsilon f^{(1)}+\varepsilon^{2} f^{(2)}+\ldots
$$

where

$$
\begin{gathered}
\operatorname{div}_{v}\left\{f\left(\alpha-\beta|v|^{2}\right) v\right\}=0 \\
\partial_{t} f+\operatorname{div}_{x}(f v)+\operatorname{div}_{v}(f a(t, x))+\operatorname{div}_{v}\left\{f^{(1)}\left(\alpha-\beta|v|^{2}\right) v\right\}=0,
\end{gathered}
$$

up to first order. Therefore, to characterize the zeroth order term in the expansion we need naturally to work with solutions whose support lies on the sphere of radius $r:=\sqrt{\alpha / \beta}$ denoted by $r \mathbb{S}$ with $\mathbb{S}=\left\{v \in \mathbb{R}^{d}:|v|=1\right\}$. In turn, we need to work with measure solutions to (44) which makes natural to set as functional space the set of nonnegative bounded Radon measures on $\mathbb{R}^{d} \times \mathbb{R}^{d}$ denoted by $\mathcal{M}_{b}^{+}\left(\mathbb{R}^{d} \times \mathbb{R}^{d}\right)$. We will be looking at solutions to (1) which are typically continuous curves in the space $\mathcal{M}_{b}^{+}\left(\mathbb{R}^{d} \times \mathbb{R}^{d}\right)$ with a suitable notion of continuity to be discussed later on. We will denote by $f^{\varepsilon}(t, x, v) \mathrm{d}(x, v)$ the integration against the measure solution $f^{\varepsilon}(t, x, v)$ of (1) at time $t$. For the sake of clarity, this is done independently of being the measure $f^{\varepsilon}(t)$ absolutely continuous with respect to Lebesgue or not, i.e., having a $L^{1}\left(\mathbb{R}^{d} \times \mathbb{R}^{d}\right)$ density or not.

Proposition 1.1 Assume that $\left(1+|v|^{2}\right) F \in \mathcal{M}_{b}^{+}\left(\mathbb{R}^{d}\right)$. Then $F$ is a solution to (4) if and only if $\operatorname{supp} F \subset\{0\} \cup r \mathbb{S}$.

The condition (4) appears as a constraint, satisfied at any time $t \in \mathbb{R}_{+}$. The time evolution of the dominant term $f$ in the Ansatz (3) will come by eliminating the multiplier $f^{(1)}$ in (5), 
provided that $f$ verifies the constraint (41). In other words we are allowed to use those test functions $\psi(x, v)$ which remove the contribution of the term $\operatorname{div}_{v}\left\{f^{(1)}\left(\alpha-\beta|v|^{2}\right) v\right\}$ i.e.,

$$
\int_{\mathbb{R}^{d} \times \mathbb{R}^{d}}\left(\alpha-\beta|v|^{2}\right) v \cdot \nabla_{v} \psi f^{(1)}(t, x, v) \mathrm{d}(x, v)=0 .
$$

Therefore we need to investigate the invariants of the field $\left(\alpha-\beta|v|^{2}\right) v \cdot \nabla_{v}$. The admissible test functions are mainly those depending on $x$ and $v /|v|, v \neq 0$. The characteristic flow $(s, v) \rightarrow \mathcal{V}(s ; v)$ associated to $\frac{1}{\varepsilon}\left(\alpha-\beta|v|^{2}\right) v \cdot \nabla_{v}$

$$
\frac{\mathrm{d} \mathcal{V}}{\mathrm{d} s}=\frac{1}{\varepsilon}\left(\alpha-\beta|\mathcal{V}(s ; v)|^{2}\right) \mathcal{V}(s ; v), \quad \mathcal{V}(0 ; v)=v
$$

will play a crucial role in our study. It will be analyzed in detail in Section 3 , Notice that the elements of $\{0\} \cup r \mathbb{S}$ are the equilibria of $\left(\alpha-\beta|v|^{2}\right) v \cdot \nabla_{v}$. It is easily seen that the jacobian of this field

$$
\partial_{v}\left\{\left(\alpha-\beta|v|^{2}\right) v\right\}=\left(\alpha-\beta|v|^{2}\right) I-2 \beta v \otimes v
$$

is negative on $r \mathbb{S}$, saying that $r \mathbb{S}$ are stable equilibria. The point 0 is unstable, $\partial_{v}\{(\alpha-$ $\left.\left.\beta|v|^{2}\right) v\right\}\left.\right|_{v=0}=\alpha I$. When $\varepsilon \searrow 0$ the solutions $\left(f^{\varepsilon}\right)_{\varepsilon}$ concentrate on $\mathbb{R}^{d} \times(\{0\} \cup r \mathbb{S})$, leading to a limit curve of measures even if $\left(f^{\varepsilon}\right)_{\varepsilon}$ were smooth solutions. We can characterize the limit curve as solution of certain PDE whenever our initial measure does not charge the unstable point 0 .

Theorem 1.1 Assume that $a \in L^{\infty}\left(\mathbb{R}_{+} ; W^{1, \infty}\left(\mathbb{R}^{d}\right)\right),\left(1+|v|^{2}\right) f^{\text {in }} \in \mathcal{M}_{b}^{+}\left(\mathbb{R}^{d} \times \mathbb{R}^{d}\right)$, supp $f^{\text {in }} \subset$ $\left\{(x, v):|v| \geq r_{0}>0\right\}$. Then $\left(f^{\varepsilon}\right)_{\varepsilon}$ converges weakly $\star$ in $L^{\infty}\left(\mathbb{R}_{+} ; \mathcal{M}_{b}\left(\mathbb{R}^{d} \times \mathbb{R}^{d}\right)\right)$ towards the solution of the problem

$$
\begin{gathered}
\partial_{t} f+\operatorname{div}_{x}(f v)+\operatorname{div}_{v}\left\{f\left(I-\frac{v \otimes v}{|v|^{2}}\right) a\right\}=0 \\
\operatorname{div}_{v}\left\{f\left(\alpha-\beta|v|^{2}\right) v\right\}=0
\end{gathered}
$$

with initial data $f(0)=\left\langle f^{\text {in }}\right\rangle$ defined by

$$
\int_{\mathbb{R}^{d} \times \mathbb{R}^{d}} \psi(x, v)\left\langle f^{\text {in }}\right\rangle(x, v) \mathrm{d}(x, v)=\int_{\mathbb{R}^{d} \times \mathbb{R}^{d}} \psi\left(x, r \frac{v}{|v|}\right) f^{\text {in }}(x, v) \mathrm{d}(x, v),
$$

for all $\psi \in C_{c}^{0}\left(\mathbb{R}^{d} \times \mathbb{R}^{d}\right)$.

In the rest, we will refer to $\left\langle f^{\text {in }}\right\rangle$ as the projected measure on the sphere of radius $r$ corresponding to $f^{\text {in }}$. Let us point out that the previous result can be equivalently written in spherical coordinates by saying that $f(t, x, \omega)$ is the measure solution to the evolution equation on $(x, \omega) \in \mathbb{R}^{d} \times r \mathbb{S}$ given by

$$
\partial_{t} f+\operatorname{div}_{x}(f \omega)+\operatorname{div}_{\omega}\left\{f\left(I-\frac{1}{r^{2}}(\omega \otimes \omega)\right) a\right\}=0
$$


These results for the linear problem, when $a(t, x, v)$ is given, can be generalized to the nonlinear counterparts where $a(t, x)$ is given by (2). The main result of this work is (see Section 2 for the definition of $\mathcal{P}_{1}$ ):

Theorem 1.2 Assume that $U \in C_{b}^{2}\left(\mathbb{R}^{d}\right), H(x, v)=h(x) v$ with $h \in C_{b}^{1}\left(\mathbb{R}^{d}\right)$ nonnegative, $f^{\text {in }} \in \mathcal{P}_{1}\left(\mathbb{R}^{d} \times \mathbb{R}^{d}\right)$, supp $f^{\text {in }} \subset\left\{(x, v):|x| \leq L_{0}, r_{0} \leq|v| \leq R_{0}\right\}$ with $0<r_{0}<r<R_{0}<\infty$. Then for all $\delta>0$, the sequence $\left(f^{\varepsilon}\right)_{\varepsilon}$ converges in $C\left([\delta, \infty) ; \mathcal{P}_{1}\left(\mathbb{R}^{d} \times \mathbb{R}^{d}\right)\right)$ towards the measure solution $f(t, x, \omega)$ on $(x, \omega) \in \mathbb{R}^{d} \times r \mathbb{S}$ of the problem

$$
\partial_{t} f+\operatorname{div}_{x}(f \omega)-\operatorname{div}_{\omega}\left\{f\left(I-\frac{1}{r^{2}}(\omega \otimes \omega)\right)\left(\nabla_{x} U \star \rho+H \star f\right)\right\}=0
$$

with initial data $f(0)=\left\langle f^{\text {in }}\right\rangle$. Moreover, if the initial data $f^{\text {in }}$ is already compactly supported on $B_{L_{0}} \times r \mathbb{S}$, then the convergence holds in $C\left(\mathbb{R}_{+} ; \mathcal{P}_{1}\left(\mathbb{R}^{d} \times \mathbb{R}^{d}\right)\right)$.

Let us mention that the evolution problem (8) on $\mathbb{R}^{d} \times r \mathbb{S}$ was also proposed in the literature as the continuum version [23] of the Vicsek model [38, 18] without diffusion for the particular choice $U=0$ and $H(x, v)=h(x) v$ with $h(x)$ some local averaging kernel. The original model in [38, 18] also includes noise at the particle level and was derived as the mean filed limit of some stochastic particle systems in [4]. In fact, previous particle systems have also been studied with noise in [3] for the mean-field limit, in [30] for studying some properties of the Cucker-Smale model with noise, and in [22, 27] for analyzing the phase transition in the Vicsek model.

In the case of noise, getting accurate control on the particle paths of the solutions is a complicated issue and thus, we are not able to show the corresponding rigorous results to Theorems 1.1 and 1.2. Nevertheless, we will present a simplified formalism, which allows us to handle more complicated problems to formally get the expected limit equations. This approach was borrowed from the framework of the magnetic confinement, where leading order charged particle densities have to be computed after smoothing out the fluctuations which correspond to the fast motion of particles around the magnetic lines [5, 6, 7, 8]. We apply this method to the following (linear or nonlinear) problem

$$
\partial_{t} f^{\varepsilon}+\operatorname{div}_{x}\left\{f^{\varepsilon} v\right\}+\operatorname{div}_{v}\left\{f^{\varepsilon} a\right\}+\frac{1}{\varepsilon} \operatorname{div}_{v}\left\{f^{\varepsilon}\left(\alpha-\beta|v|^{2}\right) v\right\}=\Delta_{v} f^{\varepsilon}
$$

with initial data $f^{\varepsilon}(0)=f^{\text {in }}$ where the acceleration $a \in L^{\infty}\left(\mathbb{R}_{+} ; W^{1, \infty}\left(\mathbb{R}^{d}\right)\right)$ and $f^{\text {in }} \in$ $\mathcal{M}_{b}^{+}\left(\mathbb{R}^{d} \times \mathbb{R}^{d}\right)$. By applying the projection operator $\langle\cdot\rangle$ to (9), we will show that the limiting equation for the evolution of $f(t, x, \omega)$ on $(x, \omega) \in \mathbb{R}^{d} \times r \mathbb{S}$ is given by

$$
\partial_{t} f+\operatorname{div}_{x}(f \omega)+\operatorname{div}_{\omega}\left\{f\left(I-\frac{1}{r^{2}}(\omega \otimes \omega)\right) a\right\}=\Delta_{\omega} f
$$


where $\Delta_{\omega}$ is the Laplace-Beltrami operator on $r \mathbb{S}$.

Our paper is organized as follows. In Section 2 we investigate the stability of the characteristic flows associated to the perturbed fields $v \cdot \nabla_{x}+a \cdot \nabla_{v}+\frac{1}{\varepsilon}\left(\alpha-\beta|v|^{2}\right) v \cdot \nabla_{v}$. The first limit result for the linear problem (cf. Theorem 1.1) is derived rigorously in Section 3 , Section 4 is devoted to the proof of the main Theorem 1.2, The new formalism to deal with the treatment of diffusion models is presented in Section 5. The computations to show that these models correspond to the Vicsek models, written in spherical coordinates, are presented in the Appendix $\mathrm{A}$.

\section{Measure solutions}

\subsection{Preliminaries on mass transportation metrics and notations}

We recall some notations and result about mass transportation distances that we will use in the sequel. For more details the reader can refer to [39, 15].

We denote by $\mathcal{P}_{1}\left(\mathbb{R}^{d}\right)$ the space of probability measures on $\mathbb{R}^{d}$ with finite first moment. We introduce the so-called Monge-Kantorovich-Rubinstein distance in $\mathcal{P}_{1}\left(\mathbb{R}^{d}\right)$ defined by

$$
W_{1}(f, g)=\sup \left\{\left|\int_{\mathbb{R}^{d}} \varphi(u)(f(u)-g(u)) \mathrm{d} u\right|, \varphi \in \operatorname{Lip}\left(\mathbb{R}^{d}\right), \operatorname{Lip}(\varphi) \leq 1\right\}
$$

where $\operatorname{Lip}\left(\mathbb{R}^{d}\right)$ denotes the set of Lipschitz functions on $\mathbb{R}^{d}$ and $\operatorname{Lip}(\varphi)$ the Lipschitz constant of a function $\varphi$. Denoting by $\Lambda$ the set of transference plans between the measures $f$ and $g$, i.e., probability measures in the product space $\mathbb{R}^{d} \times \mathbb{R}^{d}$ with first and second marginals $f$ and $g$ respectively

$$
f(y)=\int_{\mathbb{R}^{d}} \pi(y, z) \mathrm{d} z, \quad g(z)=\int_{\mathbb{R}^{d}} \pi(y, z) \mathrm{d} y
$$

then we have

$$
W_{1}(f, g)=\inf _{\pi \in \Lambda}\left\{\int_{\mathbb{R}^{d} \times \mathbb{R}^{d}}|y-z| \pi(y, z) \mathrm{d}(y, z)\right\}
$$

by Kantorovich duality. $\mathcal{P}_{1}\left(\mathbb{R}^{d}\right)$ endowed with this distance is a complete metric space. Its properties are summarized below, see[39].

Proposition 2.1 The following properties of the distance $W_{1}$ hold:

1) Optimal transference plan: The infimum in the definition of the distance $W_{1}$ is achieved. Any joint probability measure $\pi_{o}$ satisfying:

$$
W_{1}(f, g)=\int_{\mathbb{R}^{d} \times \mathbb{R}^{d}}|y-z| \mathrm{d} \pi_{o}(y, z)
$$


is called an optimal transference plan and it is generically non unique for the $W_{1}$ distance.

2) Convergence of measures: Given $\left\{f_{k}\right\}_{k \geq 1}$ and $f$ in $\mathcal{P}_{1}\left(\mathbb{R}^{d}\right)$, the following two assertions are equivalent:

a) $W_{1}\left(f_{k}, f\right)$ tends to 0 as $k$ goes to infinity.

b) $f_{k}$ tends to $f$ weakly $\star$ as measures as $k$ goes to infinity and

$$
\sup _{k \geq 1} \int_{|v|>R}|v| f_{k}(v) \mathrm{d} v \rightarrow 0 \text { as } R \rightarrow+\infty .
$$

Let us point out that if the sequence of measures is supported on a common compact set, then the convergence in $W_{1}$-sense is equivalent to standard weak- $\star$ convergence for bounded Radon measures.

Finally, let us remark that all the models considered in this paper preserve the total mass. After normalization we can consider only solutions with total mass 1 and therefore use the Monge-Kantorovich-Rubinstein distance in $\mathcal{P}_{1}\left(\mathbb{R}^{d} \times \mathbb{R}^{d}\right)$. From now on we assume that the initial conditions has total mass 1.

\subsection{Estimates on Characteristics}

In this section we investigate the linear Vlasov problem

$$
\begin{gathered}
\partial_{t} f^{\varepsilon}+\operatorname{div}_{x}\left\{f^{\varepsilon} v\right\}+\operatorname{div}_{v}\left\{f^{\varepsilon} a\right\}+\frac{1}{\varepsilon} \operatorname{div}_{v}\left\{f^{\varepsilon}\left(\alpha-\beta|v|^{2}\right) v\right\}=0, \quad(t, x, v) \in \mathbb{R}_{+} \times \mathbb{R}^{d} \times \mathbb{R}^{d} \\
f^{\varepsilon}(0)=f^{\text {in }}
\end{gathered}
$$

where $a \in L^{\infty}\left(\mathbb{R}_{+} ; W^{1, \infty}\left(\mathbb{R}^{d}\right)\right)$ and $f^{\text {in }} \in \mathcal{M}_{b}^{+}\left(\mathbb{R}^{d} \times \mathbb{R}^{d}\right)$.

Definition 2.1 Assume that $a \in L^{\infty}\left(\mathbb{R}_{+} ; W^{1, \infty}\left(\mathbb{R}^{d}\right)\right)$ and $f^{\text {in }} \in \mathcal{M}_{b}^{+}\left(\mathbb{R}^{d} \times \mathbb{R}^{d}\right)$. We say that $f^{\varepsilon} \in L^{\infty}\left(\mathbb{R}_{+} ; \mathcal{M}_{b}\left(\mathbb{R}^{d} \times \mathbb{R}^{d}\right)\right)$ is a measure solution of (11)-(12) if for any test function $\varphi \in C_{c}^{1}\left(\mathbb{R}_{+} \times \mathbb{R}^{d} \times \mathbb{R}^{d}\right)$ we have

$$
\begin{aligned}
\int_{\mathbb{R}_{+}} \int_{\mathbb{R}^{d} \times \mathbb{R}^{d}}\left\{\partial_{t}+v \cdot \nabla_{x}+a \cdot \nabla_{v}+\frac{1}{\varepsilon}\left(\alpha-\beta|v|^{2}\right) v\right. & \left.\cdot \nabla_{v}\right\} \varphi f^{\varepsilon}(t, x, v) \mathrm{d}(x, v) \mathrm{d} t \\
& +\int_{\mathbb{R}^{d} \times \mathbb{R}^{d}} \varphi(0, x, v) f^{\mathrm{in}}(x, v) \mathrm{d}(x, v)=0 .
\end{aligned}
$$

We introduce the characteristics of the field $v \cdot \nabla_{x}+a \cdot \nabla_{v}+\frac{1}{\varepsilon}\left(\alpha-\beta|v|^{2}\right) v \cdot \nabla_{v}$

$$
\frac{\mathrm{d} X^{\varepsilon}}{\mathrm{d} s}=V^{\varepsilon}(s), \quad \frac{\mathrm{d} V^{\varepsilon}}{\mathrm{d} s}=a\left(s, X^{\varepsilon}(s)\right)+\frac{1}{\varepsilon}\left(\alpha-\beta\left|V^{\varepsilon}(s)\right|^{2}\right) V^{\varepsilon}(s)
$$




$$
X^{\varepsilon}(s=0)=x, \quad V^{\varepsilon}(s=0)=v .
$$

We will prove that $\left(X^{\varepsilon}, V^{\varepsilon}\right)$ are well defined for any $(s, x, v) \in \mathbb{R}_{+} \times \mathbb{R}^{d} \times \mathbb{R}^{d}$. Indeed, on any interval $[0, T]$ on which $\left(X^{\varepsilon}, V^{\varepsilon}\right)$ is well defined we get a bound

$$
\sup _{s \in[0, T]}\left\{\left|X^{\varepsilon}(s)\right|+\left|V^{\varepsilon}(s)\right|\right\}<+\infty
$$

implying that the characteristics are global in positive time. For that we write

$$
\frac{1}{2} \frac{\mathrm{d}\left|V^{\varepsilon}\right|^{2}}{\mathrm{~d} s}=a\left(s, X^{\varepsilon}(s)\right) \cdot V^{\varepsilon}(s)+\frac{1}{\varepsilon}\left(\alpha-\beta\left|V^{\varepsilon}(s)\right|^{2}\right)\left|V^{\varepsilon}(s)\right|^{2} .
$$

and then, we get the differential inequality

$$
\frac{\mathrm{d}\left|V^{\varepsilon}\right|^{2}}{\mathrm{~d} s} \leq 2\|a\|_{L^{\infty}}\left|V^{\varepsilon}(s)\right|+\frac{2}{\varepsilon}\left(\alpha-\beta\left|V^{\varepsilon}(s)\right|^{2}\right)\left|V^{\varepsilon}(s)\right|^{2}
$$

for all $s \in[0, T]$, so that

$$
\sup _{s \in[0, T]}\left|V^{\varepsilon}(s)\right|<+\infty, \sup _{s \in[0, T]}\left|X^{\varepsilon}(s)\right| \leq|x|+T \sup _{s \in[0, T]}\left|V^{\varepsilon}(s)\right|<+\infty .
$$

Once constructed the characteristics, it is easily seen how to obtain a measure solution for the Vlasov problem (11)-(12). It reduces to push forward the initial measure along the characteristics, see [10] for instance.

Proposition 2.2 For any $t \in \mathbb{R}_{+}$we denote by $f^{\varepsilon}(t)$ the measure given by

$$
\int_{\mathbb{R}^{d} \times \mathbb{R}^{d}} \psi(x, v) f^{\varepsilon}(t, x, v) \mathrm{d}(x, v)=\int_{\mathbb{R}^{d} \times \mathbb{R}^{d}} \psi\left(\left(X^{\varepsilon}, V^{\varepsilon}\right)(t ; 0, x, v)\right) f^{\mathrm{in}}(x, v) \mathrm{d}(x, v),
$$

for all $\psi \in C_{c}^{0}\left(\mathbb{R}^{d} \times \mathbb{R}^{d}\right)$. Then the application $t \rightarrow f^{\varepsilon}(t)$, denoted $f^{\text {in }} \#\left(X^{\varepsilon}, V^{\varepsilon}\right)(t ; 0, \cdot, \cdot)$ is the unique measure solution of (11), (12), belongs to $C\left(\mathbb{R}_{+} ; \mathcal{M}_{b}\left(\mathbb{R}^{d} \times \mathbb{R}^{d}\right)\right)$ and satisfies

$$
\int_{\mathbb{R}^{d} \times \mathbb{R}^{d}} f^{\varepsilon}(t, x, v) \mathrm{d}(x, v)=\int_{\mathbb{R}^{d} \times \mathbb{R}^{d}} f^{\mathrm{in}}(x, v) \mathrm{d}(x, v), t \in \mathbb{R}_{+} .
$$

Proof. The arguments are straightforward and are left to the reader. We only justify that $f^{\varepsilon} \in C\left(\mathbb{R}_{+} ; \mathcal{M}_{b}\left(\mathbb{R}^{d} \times \mathbb{R}^{d}\right)\right)$ meaning that for any $\psi \in C_{c}^{0}\left(\mathbb{R}^{d} \times \mathbb{R}^{d}\right)$ the application $t \rightarrow \int_{\mathbb{R}^{d} \times \mathbb{R}^{d}} \psi(x, v) f^{\varepsilon}(t, x, v) \mathrm{d}(x, v)$ is continuous. Choose $\psi \in C_{c}^{0}\left(\mathbb{R}^{d} \times \mathbb{R}^{d}\right)$. Then, for any $0 \leq t_{1}<t_{2}$ we have

$$
\begin{aligned}
\int_{\mathbb{R}^{d} \times \mathbb{R}^{d}} \psi(x, v) f^{\varepsilon}\left(t_{2}, x, v\right) \mathrm{d}(x, v)-\int_{\mathbb{R}^{d} \times \mathbb{R}^{d}} \psi(x, v) f^{\varepsilon}\left(t_{1}, x, v\right) \mathrm{d}(x, v) \\
=\int_{\mathbb{R}^{d} \times \mathbb{R}^{d}}\left[\psi\left(\left(X^{\varepsilon}, V^{\varepsilon}\right)\left(t_{2} ; t_{1}, x, v\right)\right)-\psi(x, v)\right] f^{\varepsilon}\left(t_{1}, x, v\right) \mathrm{d}(x, v) .
\end{aligned}
$$


Taking into account that $\left(X^{\varepsilon}, V^{\varepsilon}\right)$ are locally bounded (in time, position, velocity) it is easily seen that for any compact set $K \subset \mathbb{R}^{d} \times \mathbb{R}^{d}$ there is a constant $C(K)$ such that

$$
\left|X^{\varepsilon}\left(t_{2} ; t_{1}, x, v\right)-x\right|+\left|V^{\varepsilon}\left(t_{2} ; t_{1}, x, v\right)-v\right| \leq\left|t_{2}-t_{1}\right| C(K), \quad(x, v) \in K .
$$

Our conclusion follows easily using the uniform continuity of $\psi$ and that $\left\|f^{\varepsilon}\left(t_{1}\right)\right\|_{\mathcal{M}_{b}}=$ $\left\|f^{\text {in }}\right\|_{\mathcal{M}_{b}}$. Notice also that the equality (14) holds true for any bounded continuous function $\psi$.

We intend to study the behavior of $\left(f^{\varepsilon}\right)_{\varepsilon}$ when $\varepsilon$ becomes small. This will require a more detailed analysis of the characteristic flows $\left(X^{\varepsilon}, V^{\varepsilon}\right)$. The behavior of these characteristics depends on the roots of functions like $A+\frac{1}{\varepsilon}\left(\alpha-\beta \rho^{2}\right) \rho$, with $\rho \in \mathbb{R}_{+}, A \in \mathbb{R}$.

Proposition 2.3 Assume that $A<0$ and $0<\varepsilon<2 \alpha r /(|A| 3 \sqrt{3})$. Then the equation $\lambda^{\varepsilon}(\rho):=\varepsilon A+\left(\alpha-\beta \rho^{2}\right) \rho=0$ has two zeros on $\mathbb{R}_{+}$, denoted $\rho_{1}^{\varepsilon}(A), \rho_{2}^{\varepsilon}(A)$, satisfying

$$
0<\rho_{1}^{\varepsilon}<\frac{r}{\sqrt{3}}<\rho_{2}^{\varepsilon}<r
$$

and

$$
\lim _{\varepsilon \searrow 0} \frac{\rho_{1}^{\varepsilon}}{\varepsilon}=\frac{|A|}{\alpha}, \quad \lim _{\varepsilon \searrow 0} \frac{r-\rho_{2}^{\varepsilon}}{\varepsilon}=\frac{|A|}{2 \alpha}
$$

where $r=\sqrt{\alpha / \beta}$.

Proof. It is easily seen that the function $\lambda^{\varepsilon}$ increases on $[0, r / \sqrt{3}]$ and decreases on $\left[r / \sqrt{3},+\infty\left[\right.\right.$ with change of sign on $[0, r / \sqrt{3}]$ and $[r / \sqrt{3}, r]$. We can prove that $\left(\rho_{1}^{\varepsilon}\right)_{\varepsilon},\left(\rho_{2}^{\varepsilon}\right)_{\varepsilon}$ are monotone with respect to $\varepsilon>0$. Take $0<\varepsilon<\bar{\varepsilon}<2 \alpha r /(|A| 3 \sqrt{3})$ and observe that $\lambda^{\varepsilon}>\lambda^{\bar{\varepsilon}}$. In particular we have

$$
\lambda^{\bar{\varepsilon}}\left(\rho_{1}^{\varepsilon}\right)<\lambda^{\varepsilon}\left(\rho_{1}^{\varepsilon}\right)=0=\lambda^{\bar{\varepsilon}}\left(\rho_{1}^{\bar{\varepsilon}}\right)
$$

implying $\rho_{1}^{\varepsilon}<\rho_{1}^{\bar{\varepsilon}}$, since $\lambda^{\bar{\varepsilon}}$ is strictly increasing on $[0, r / \sqrt{3}]$. Similarly we have

$$
\lambda^{\bar{\varepsilon}}\left(\rho_{2}^{\varepsilon}\right)<\lambda^{\varepsilon}\left(\rho_{2}^{\varepsilon}\right)=0<\lambda^{\bar{\varepsilon}}\left(\rho_{2}^{\bar{\varepsilon}}\right)
$$

and thus $\rho_{2}^{\varepsilon}>\rho_{2}^{\bar{\varepsilon}}$, since $\lambda^{\bar{\varepsilon}}$ is strictly decreasing on $[r / \sqrt{3}, r]$. Passing to the limit in $\lambda^{\varepsilon}\left(\rho_{k}^{\varepsilon}\right)=$ $0, k \in\{1,2\}$ it follows easily that

$$
\lim _{\varepsilon \searrow 0} \rho_{1}^{\varepsilon}=0, \lim _{\varepsilon \searrow 0} \rho_{2}^{\varepsilon}=r
$$

Moreover we can write

$$
\alpha=\left.\frac{\mathrm{d}}{\mathrm{d} \rho}\left\{\left(\alpha-\beta \rho^{2}\right) \rho\right\}\right|_{\rho=0}=\lim _{\varepsilon \searrow 0} \frac{\left[\alpha-\beta\left(\rho_{1}^{\varepsilon}\right)^{2}\right] \rho_{1}^{\varepsilon}}{\rho_{1}^{\varepsilon}}=-\lim _{\varepsilon \searrow 0} \frac{\varepsilon A}{\rho_{1}^{\varepsilon}}
$$


and

$$
-2 \alpha=\left.\frac{\mathrm{d}}{\mathrm{d} \rho}\left\{\left(\alpha-\beta \rho^{2}\right) \rho\right\}\right|_{\rho=r}=\lim _{\varepsilon \searrow 0} \frac{\left[\alpha-\beta\left(\rho_{2}^{\varepsilon}\right)^{2}\right] \rho_{2}^{\varepsilon}}{\rho_{2}^{\varepsilon}-r}=-\lim _{\varepsilon \searrow 0} \frac{\varepsilon A}{\rho_{2}^{\varepsilon}-r}
$$

saying that

$$
\lim _{\varepsilon \searrow 0} \frac{\rho_{1}^{\varepsilon}}{\varepsilon}=\frac{|A|}{\alpha}, \lim _{\varepsilon \searrow 0} \frac{r-\rho_{2}^{\varepsilon}}{\varepsilon}=\frac{|A|}{2 \alpha} .
$$

The case $A>0$ can be treated is a similar way and we obtain

Proposition 2.4 Assume that $A>0$ and $\varepsilon>0$. Then the equation $\lambda^{\varepsilon}(\rho):=\varepsilon A+(\alpha-$ $\left.\beta \rho^{2}\right) \rho=0$ has one zero on $\mathbb{R}_{+}$, denoted $\rho_{3}^{\varepsilon}(A)$, satisfying

$$
\rho_{3}^{\varepsilon}>r, \lim _{\varepsilon \searrow 0} \frac{\rho_{3}^{\varepsilon}-r}{\varepsilon}=\frac{|A|}{2 \alpha} .
$$

Using the sign of the function $\rho \rightarrow \varepsilon\|a\|_{L^{\infty}}+\left(\alpha-\beta \rho^{2}\right) \rho$ we obtain the following bound for the kinetic energy.

Proposition 2.5 Assume that $a \in L^{\infty}\left(\mathbb{R}_{+} ; W^{1, \infty}\left(\mathbb{R}^{d}\right)\right),\left(1+|v|^{2}\right) f^{\text {in }} \in \mathcal{M}_{b}^{+}\left(\mathbb{R}^{d} \times \mathbb{R}^{d}\right)$ and let us denote by $f^{\varepsilon}$ the unique measure solution of (111), (12). Then we have

$$
\left\|\int_{\mathbb{R}^{d} \times \mathbb{R}^{d}}|v|^{2} f^{\varepsilon}(\cdot, x, v) \mathrm{d}(x, v)\right\|_{L^{\infty}\left(\mathbb{R}_{+}\right)} \leq \int_{\mathbb{R}^{d} \times \mathbb{R}^{d}}\left[\left(\rho_{3}^{\varepsilon}\right)^{2}+|v|^{2}\right] f^{\mathrm{in}}(x, v) \mathrm{d}(x, v) .
$$

Proof. We know that

$$
\frac{\mathrm{d}}{\mathrm{d} t}\left|V^{\varepsilon}\right|^{2} \leq 2\|a\|_{L^{\infty}}\left|V^{\varepsilon}(t)\right|+\frac{2}{\varepsilon}\left(\alpha-\beta\left|V^{\varepsilon}(t)\right|^{2}\right)\left|V^{\varepsilon}(t)\right|^{2}=\frac{2}{\varepsilon}\left|V^{\varepsilon}(t)\right| \lambda^{\varepsilon}\left(\left|V^{\varepsilon}(\bar{t})\right|\right), \quad t \in \mathbb{R}_{+} .
$$

By comparison with the solutions of the autonomous differential equation associated to the righthand side, we easily deduce that

$$
\left|V^{\varepsilon}(t ; 0, x, v)\right| \leq \max \left\{|v|, \rho_{3}^{\varepsilon}\left(\|a\|_{L^{\infty}}\right)\right\}
$$

for any $T \in \mathbb{R}_{+},(x, v) \in \mathbb{R}^{d} \times \mathbb{R}^{d}$. This yields the following bound for the kinetic energy

$$
\begin{aligned}
\int_{\mathbb{R}^{d} \times \mathbb{R}^{d}}|v|^{2} f^{\varepsilon}(T, x, v) \mathrm{d}(x, v) & =\int_{\mathbb{R}^{d} \times \mathbb{R}^{d}}\left|V^{\varepsilon}(T ; 0, x, v)\right|^{2} f^{\mathrm{in}}(x, v) \mathrm{d}(x, v) \\
& \leq \int_{\mathbb{R}^{d} \times \mathbb{R}^{d}}\left[\left(\rho_{3}^{\varepsilon}\right)^{2}+|v|^{2}\right] f^{\mathrm{in}}(x, v) \mathrm{d}(x, v) .
\end{aligned}
$$

The object of the next result is to establish the stability of $V^{\varepsilon}$ around $|v|=r$. We will show that the characteristics starting at points with velocities inside an annulus of length proportional to $\varepsilon$ around the sphere $r \mathbb{S}$ get trapped there for all positive times for small $\varepsilon$. 
Proposition 2.6 Assume that $\varepsilon\|a\|_{L^{\infty}}<2 \alpha r /(3 \sqrt{3})$ and that $\rho_{2}^{\varepsilon}\left(-\|a\|_{L^{\infty}}\right) \leq|v| \leq \rho_{3}^{\varepsilon}\left(\|a\|_{L^{\infty}}\right)$. Then, for any $(t, x) \in \mathbb{R}_{+} \times \mathbb{R}^{d}$ we have

$$
\rho_{2}^{\varepsilon}\left(-\|a\|_{L^{\infty}}\right) \leq\left|V^{\varepsilon}(t ; 0, x, v)\right| \leq \rho_{3}^{\varepsilon}\left(\|a\|_{L^{\infty}}\right) .
$$

Proof. As in previous proof, we know that

$$
\frac{\mathrm{d}}{\mathrm{d} t}\left|V^{\varepsilon}\right|^{2} \leq \frac{2}{\varepsilon}\left|V^{\varepsilon}(t)\right| \lambda^{\varepsilon}\left(\left|V^{\varepsilon}(\bar{t})\right|\right), \quad t \in \mathbb{R}_{+} .
$$

By comparison with the constant solution $\rho_{3}^{\varepsilon}$ to the autonomous differential equation associated to the righthand side, we get that $\sup _{t \in \mathbb{R}_{+}}\left|V^{\varepsilon}(t ; 0, x, v)\right| \leq \rho_{3}^{\varepsilon}$. Assume now that there is $T>0$ such that $\left|V^{\varepsilon}(T)\right|<\rho_{2}^{\varepsilon}$ and we are done if we find a contradiction. Since $\left|V^{\varepsilon}(0)\right|=|v| \geq \rho_{2}^{\varepsilon}$, we can assume that $\min _{t \in[0, T]}\left|V^{\varepsilon}(t)\right|>\rho_{1}^{\varepsilon}>0$ by time continuity. Take now $\bar{t} \in[0, T]$ a minimum point of $t \rightarrow\left|V^{\varepsilon}(t)\right|$ on $[0, T]$. Obviously $\bar{t}>0$ since

$$
\left|V^{\varepsilon}(\bar{t})\right| \leq\left|V^{\varepsilon}(T)\right|<\rho_{2}^{\varepsilon} \leq|v|=\left|V^{\varepsilon}(0)\right| .
$$

By estimating from below in (13) and using that $\bar{t}$ is a minimum point of $t \rightarrow\left|V^{\varepsilon}(t)\right|>0$ on $[0, T]$, we obtain

$$
0 \geq \frac{\mathrm{d}}{\mathrm{d} t}\left|V^{\varepsilon}(\bar{t})\right| \geq-\|a\|_{L^{\infty}}+\frac{\left(\alpha-\beta\left|V^{\varepsilon}(\bar{t})\right|^{2}\right)\left|V^{\varepsilon}(\bar{t})\right|}{\varepsilon}=\frac{\lambda^{\varepsilon}\left(\left|V^{\varepsilon}(\bar{t})\right|\right)}{\varepsilon} .
$$

But the function $\lambda^{\varepsilon}$ has negative sign on $\left[0, \rho_{1}^{\varepsilon}\right] \cup\left[\rho_{2}^{\varepsilon},+\infty\right.$. Since we know that $\min _{t \in[0, T]}\left|V^{\varepsilon}(t)\right|>$ $\rho_{1}^{\varepsilon}$, it remains that

$$
\min _{t \in[0, T]}\left|V^{\varepsilon}(t)\right|=\left|V^{\varepsilon}(\bar{t})\right| \geq \rho_{2}^{\varepsilon}
$$

which contradicts the assumption $\left|V^{\varepsilon}(T)\right|<\rho_{2}^{\varepsilon}$.

Let us see now what happens when the initial velocity is outside $\left[\rho_{2}^{\varepsilon}\left(-\|a\|_{L^{\infty}}\right), \rho_{3}^{\varepsilon}\left(\|a\|_{L^{\infty}}\right)\right]$. In particular we prove that if initially $v \neq 0$, then $V^{\varepsilon}(t), t \in \mathbb{R}_{+}$remains away from 0 . We actually show that the characteristics starting away from zero speed but inside the sphere $r \mathbb{S}$ will increase their speed with respect to its initial value while those starting with a speed outside the sphere $r \mathbb{S}$ will decrease their speed with respect to its initial value, all for sufficiently small $\varepsilon$.

Proposition 2.7 Consider $\varepsilon>0$ such that $\varepsilon\|a\|_{L^{\infty}}<2 \alpha r /(3 \sqrt{3})$.

1. Assume that $\rho_{1}^{\varepsilon}\left(-\|a\|_{L^{\infty}}\right)<|v|<\rho_{2}^{\varepsilon}\left(-\|a\|_{L^{\infty}}\right)$. Then for any $(t, x) \in \mathbb{R}_{+}^{\star} \times \mathbb{R}^{d}$ we have

$$
\rho_{1}^{\varepsilon}\left(-\|a\|_{L^{\infty}}\right)<|v|<\left|V^{\varepsilon}(t ; 0, x, v)\right| \leq \rho_{3}^{\varepsilon}\left(\|a\|_{L^{\infty}}\right) .
$$

2. Assume that $\rho_{3}^{\varepsilon}\left(\|a\|_{L^{\infty}}\right)<|v|$. Then for any $(t, x) \in \mathbb{R}_{+}^{\star} \times \mathbb{R}^{d}$ we have

$$
\rho_{2}^{\varepsilon}\left(-\|a\|_{L^{\infty}}\right) \leq\left|V^{\varepsilon}(t ; 0, x, v)\right|<|v| .
$$


Proof. 1. Notice that if $\left|V^{\varepsilon}(T ; 0, x, v)\right|=\rho_{2}^{\varepsilon}$ for some $T>0$, then we deduce by Proposition 2.6 that $\rho_{2}^{\varepsilon} \leq\left|V^{\varepsilon}(t)\right| \leq \rho_{3}^{\varepsilon}$ for any $t>T$ and thus $\left|V^{\varepsilon}(t ; 0, x, v)\right| \geq \rho_{2}^{\varepsilon}>|v|, t \geq T$. It remains to establish our statement for intervals $[0, T]$ such that $\left|V^{\varepsilon}(t)\right|<\rho_{2}^{\varepsilon}$ for any $t \in[0, T]$. We are done if we prove that $t \rightarrow\left|V^{\varepsilon}(t)\right|$ is strictly increasing on $[0, T]$. For any $\left.\left.\tau \in\right] 0, T\right]$ let us denote by $\bar{t}$ a maximum point of $t \rightarrow\left|V^{\varepsilon}(t)\right|>0$ on $[0, \tau]$. If $\bar{t} \in\left[0, \tau\left[\right.\right.$ we have $\frac{\mathrm{d}}{\mathrm{d} t}\left|V^{\varepsilon}(\bar{t})\right| \leq 0$ and thus

$$
0 \geq \frac{\mathrm{d}}{\mathrm{d} t}\left|V^{\varepsilon}(\bar{t})\right| \geq-\|a\|_{L^{\infty}}+\frac{\left(\alpha-\beta\left|V^{\varepsilon}(\bar{t})\right|^{2}\right)\left|V^{\varepsilon}(\bar{t})\right|}{\varepsilon}=\frac{\lambda^{\varepsilon}\left(\left|V^{\varepsilon}(\bar{t})\right|\right)}{\varepsilon} .
$$

By construction $\left|V^{\varepsilon}(\bar{t})\right|<\rho_{2}^{\varepsilon}$ and moreover,

$$
\left|V^{\varepsilon}(\bar{t})\right|=\max _{[0, \tau]}\left|V^{\varepsilon}\right| \geq|v|>\rho_{1}^{\varepsilon},
$$

and thus, $\lambda^{\varepsilon}\left(\left|V^{\varepsilon}(t)\right|\right)>0$ for all $t \in[0, T]$. Consequently, we infer that $t \rightarrow\left|V^{\varepsilon}(t)\right|$ is strictly increasing on $[0, T]$ since

$$
\frac{\mathrm{d}}{\mathrm{d} t}\left|V^{\varepsilon}(t)\right| \geq-\|a\|_{L^{\infty}}+\frac{\left(\alpha-\beta\left|V^{\varepsilon}(t)\right|^{2}\right)\left|V^{\varepsilon}(t)\right|}{\varepsilon}=\frac{\lambda^{\varepsilon}\left(\left|V^{\varepsilon}(t)\right|\right)}{\varepsilon}>0 .
$$

Therefore we have $\bar{t}=\tau$ saying that $\left|V^{\varepsilon}(\tau)\right| \geq|v|$ for any $\tau \in[0, T]$.

2. As before, it is sufficient to work on intervals $[0, T]$ such that $\left|V^{\varepsilon}(t)\right|>\rho_{3}^{\varepsilon}\left(\|a\|_{L^{\infty}}\right)$ for any $t \in[0, T]$. We are done if we prove that $t \rightarrow\left|V^{\varepsilon}(t)\right|$ is strictly decreasing on $[0, T]$. We have for any $t \in[0, T]$

$$
\frac{\mathrm{d}}{\mathrm{d} t}\left|V^{\varepsilon}(t)\right| \leq\|a\|_{L^{\infty}}+\frac{\left(\alpha-\beta\left|V^{\varepsilon}(t)\right|^{2}\right)\left|V^{\varepsilon}(t)\right|}{\varepsilon}=\frac{\lambda^{\varepsilon}\left(\left|V^{\varepsilon}(t)\right|\right)}{\varepsilon}<0
$$

where for the last inequality we have used $\left|V^{\varepsilon}(t)\right|>\rho_{3}^{\varepsilon}, t \in[0, T]$.

\section{The limit model}

We investigate now the stability of the family $\left(f^{\varepsilon}\right)_{\varepsilon}$ when $\varepsilon$ becomes small. After extraction of a sequence $\left(\varepsilon_{k}\right)_{k}$ converging to 0 we can assume that $\left(f^{\varepsilon_{k}}\right)_{k}$ converges weakly $\star$ in $L^{\infty}\left(\mathbb{R}_{+} ; \mathcal{M}_{b}\left(\mathbb{R}^{d} \times \mathbb{R}^{d}\right)\right)$, meaning that

$$
\lim _{k \rightarrow+\infty} \int_{\mathbb{R}_{+}} \int_{\mathbb{R}^{d} \times \mathbb{R}^{d}} \varphi(t, x, v) f^{\varepsilon_{k}}(t, x, v) \mathrm{d}(x, v) \mathrm{d} t=\int_{\mathbb{R}_{+}} \int_{\mathbb{R}^{d} \times \mathbb{R}^{d}} \varphi(t, x, v) f(t, x, v) \mathrm{d}(x, v) \mathrm{d} t
$$

for any $\varphi \in L^{1}\left(\mathbb{R}_{+} ; C_{c}^{0}\left(\mathbb{R}^{d} \times \mathbb{R}^{d}\right)\right)$. Using the weak formulation of (11)-(12) with test functions $\eta(t) \varphi(x, v), \eta \in C_{c}^{1}\left(\mathbb{R}_{+}\right), \varphi \in C_{c}^{1}\left(\mathbb{R}^{d} \times \mathbb{R}^{d}\right)$ one gets

$$
\begin{array}{r}
\int_{\mathbb{R}_{+}} \int_{\mathbb{R}^{d} \times \mathbb{R}^{d}}\left\{\eta^{\prime}(t) \varphi+\eta(t) v \cdot \nabla_{x} \varphi+\eta(t) a \cdot \nabla_{v} \varphi\right\} f^{\varepsilon_{k}}(t, x, v) \mathrm{d}(x, v) \mathrm{d} t \\
+\frac{1}{\varepsilon_{k}} \int_{\mathbb{R}_{+}} \int_{\mathbb{R}^{d} \times \mathbb{R}^{d}} \eta(t)\left(\alpha-\beta|v|^{2}\right) v \cdot \nabla_{v} \varphi f^{\varepsilon_{k}}(t, x, v) \mathrm{d}(x, v) \mathrm{d} t \\
=-\int_{\mathbb{R}^{d} \times \mathbb{R}^{d}} \eta(0) \varphi(x, v) f^{\mathrm{in}}(x, v) \mathrm{d}(x, v) .
\end{array}
$$


Multiplying by $\varepsilon_{k}$ and passing to the limit for $k \rightarrow+\infty$ yields

$$
\int_{\mathbb{R}_{+}} \int_{\mathbb{R}^{d} \times \mathbb{R}^{d}} \eta(t)\left(\alpha-\beta|v|^{2}\right) v \cdot \nabla_{v} \varphi f(t, x, v) \mathrm{d}(x, v) \mathrm{d} t=0
$$

and therefore one gets for any $t \in \mathbb{R}_{+}$and $\varphi \in C_{c}^{1}\left(\mathbb{R}^{d} \times \mathbb{R}^{d}\right)$

$$
\int_{\mathbb{R}^{d} \times \mathbb{R}^{d}}\left(\alpha-\beta|v|^{2}\right) v \cdot \nabla_{v} \varphi f(t, x, v) \mathrm{d}(x, v)=0 .
$$

Under the hypothesis $\left(1+|v|^{2}\right) f^{\text {in }} \in \mathcal{M}_{b}^{+}\left(\mathbb{R}^{d} \times \mathbb{R}^{d}\right)$ we deduce by Proposition 2.5 that $(1+$ $\left.|v|^{2}\right) f(t) \in \mathcal{M}_{b}^{+}\left(\mathbb{R}^{d} \times \mathbb{R}^{d}\right)$ and therefore, applying the $(x, v)$ version of Proposition 1.1 (whose proof is detailed in the sequel), we obtain

$$
\operatorname{supp} f(t) \subset \mathbb{R}^{d} \times(\{0\} \cup r \mathbb{S}), \quad t \in \mathbb{R}_{+} .
$$

The proof of Proposition 1.1 is based on the resolution of the adjoint problem

$$
-\left(\alpha-\beta|v|^{2}\right) v \cdot \nabla_{v} \varphi=\psi(v), \quad v \in \mathbb{R}^{d}
$$

for any smooth righthand side $\psi$ with compact support in ${ }^{c}(\{0\} \cup r \mathbb{S})$.

Proof. (of Proposition 1.1) It is easily seen that for any $F \in \mathcal{M}_{b}^{+}\left(\mathbb{R}^{d} \times \mathbb{R}^{d}\right.$ ), supp $F \subset\{0\} \cup r \mathbb{S}$ and any $\varphi \in C_{c}^{1}\left(\mathbb{R}^{d}\right)$ we have

$$
\int_{\mathbb{R}^{d}}\left(\alpha-\beta|v|^{2}\right) v \cdot \nabla_{v} \varphi(v) F(v) \mathrm{d} v=0
$$

saying that $\operatorname{div}_{v}\left\{F\left(\alpha-\beta|v|^{2}\right) v\right\}=0$. Assume now that $\operatorname{div}_{v}\left\{F\left(\alpha-\beta|v|^{2}\right) v\right\}=0$ for some $F \in \mathcal{M}_{b}^{+}\left(\mathbb{R}^{d} \times \mathbb{R}^{d}\right)$ and let us prove that supp $F \subset\{0\} \cup r \mathbb{S}$. We introduce the flow $\mathcal{V}=\mathcal{V}(s ; v)$ given by

$$
\frac{\mathrm{d} \mathcal{V}}{\mathrm{d} s}=\left(\alpha-\beta|\mathcal{V}(s ; v)|^{2}\right) \mathcal{V}(s ; v), \quad \mathcal{V}(0 ; v)=v .
$$

A direct computation shows that $\frac{v}{|v|}$ are left invariant

$$
\left(\alpha-\beta|v|^{2}\right) v \cdot \nabla_{v}\left(\frac{v}{|v|}\right)=\left(\alpha-\beta|v|^{2}\right)\left(I-\frac{v \otimes v}{|v|^{2}}\right) \frac{v}{|v|}=0
$$

and therefore

$$
\mathcal{V}(s ; v)=|\mathcal{V}(s ; v)| \frac{v}{|v|}, \quad v \neq 0 .
$$

Multiplying (15) by $\mathcal{V}(s ; v) /|\mathcal{V}(s ; v)|$ yields

$$
\frac{\mathrm{d}}{\mathrm{d} s}|\mathcal{V}|=\left(\alpha-\beta|\mathcal{V}(s ; v)|^{2}\right)|\mathcal{V}(s ; v)|
$$

whose solution is given by

$$
|\mathcal{V}(s ; v)|=|v| \frac{r e^{\alpha s}}{\sqrt{|v|^{2}\left(e^{2 \alpha s}-1\right)+r^{2}}}
$$


Finally one gets

$$
\left.\mathcal{V}(s ; v)=\frac{r e^{\alpha s}}{\sqrt{|v|^{2}\left(e^{2 \alpha s}-1\right)+r^{2}}} v, s \in\right] S(v),+\infty[
$$

with $S(v)=-\infty$ if $0 \leq|v| \leq r$ and $S(v)=\frac{1}{2 \alpha} \ln \left(1-\frac{r^{2}}{|v|^{2}}\right)<0$ if $|v|>r$. Notice that the characteristics $\mathcal{V}(\cdot ; v)$ are well defined on $\mathbb{R}_{+}$for any $v \in \mathbb{R}^{d}$ and we have

$$
\lim _{s \rightarrow+\infty} \mathcal{V}(s ; v)=r \frac{v}{|v|} \text { if } v \neq 0, \lim _{s \rightarrow+\infty} \mathcal{V}(s ; v)=0 \text { if } v=0
$$

and

$$
\lim _{s \searrow S(v)}|\mathcal{V}(s)|=0 \text { if } 0 \leq|v|<r, \lim _{s \searrow S(v)}|\mathcal{V}(s)|=r \text { if }|v|=r, \lim _{s \searrow S(v)}|\mathcal{V}(s)|=+\infty \text { if }|v|>r .
$$

Let us consider a $C^{1}$ function $\psi=\psi(v)$ with compact support in ${ }^{c}(\{0\} \cup r \mathbb{S})$. We intend to construct a bounded $C^{1}$ function $\varphi=\varphi(v)$ such that

$$
-\left(\alpha-\beta|v|^{2}\right) v \cdot \nabla_{v} \varphi=\psi(v), \quad v \in \mathbb{R}^{d} .
$$

Obviously, if such a function exists, we may assume that $\varphi(0)=0$. Motivated by the equality

$$
-\frac{\mathrm{d}}{\mathrm{d} s}\{\varphi(\mathcal{V}(s ; v))\}=\psi(\mathcal{V}(s ; v)), \quad 0 \leq|v|<r, \quad-\infty<s \leq 0
$$

and since we know that $\lim _{s \rightarrow-\infty} \mathcal{V}(s ; v)=0$ for any $0 \leq|v|<r$, we define

$$
\varphi(v)=-\int_{-\infty}^{0} \psi(\mathcal{V}(\tau ; v)) \mathrm{d} \tau, \quad 0 \leq|v|<r
$$

Let us check that the function $\varphi$ in (16) is well defined and is $C^{1}$ in $|v|<r$. The key point is that $\psi$ has compact support in ${ }^{c}(\{0\} \cup r \mathbb{S})$ and therefore there are $0<r_{1}<r_{2}<r<r_{3}<$ $r_{4}<+\infty$ such that $\operatorname{supp} \psi \subset\left\{v \in \mathbb{R}^{d}: r_{1} \leq|v| \leq r_{2}\right\} \cup\left\{v \in \mathbb{R}^{d}: r_{3} \leq|v| \leq r_{4}\right\}$. It is easily seen that $\tau \rightarrow|\mathcal{V}(\tau ; v)|$ is strictly increasing for any $0<|v|<r$. Therefore, for any $|v| \leq r_{1}$ we have $|\mathcal{V}(\tau ; v)| \leq|\mathcal{V}(0 ; v)|=|v| \leq r_{1}, \quad \tau \leq 0$, implying that

$$
\varphi(v)=-\int_{-\infty}^{0} \psi(\mathcal{V}(\tau ; v)) \mathrm{d} \tau=0, \quad 0 \leq|v| \leq r_{1}
$$

For any $v$ with $r_{1}<|v|<r_{2}$ there are $\tau_{1}<0<\tau_{2}$ such that $\left|\mathcal{V}\left(\tau_{1} ; v\right)\right|=r_{1}<r_{2}=\left|\mathcal{V}\left(\tau_{2} ; v\right)\right|$. The time interval between $\tau_{1}$ and $\tau_{2}$ comes easily by writing

$$
\frac{\frac{\mathrm{d}}{\mathrm{d} \tau}|\mathcal{V}(\tau)|}{\left(\alpha-\beta|\mathcal{V}(\tau)|^{2}\right)|\mathcal{V}(\tau)|}=1
$$

implying that

$$
\left|\tau_{2}\right|+\left|\tau_{1}\right|=\tau_{2}-\tau_{1}=\int_{r_{1}}^{r_{2}} \frac{\mathrm{d} \rho}{\left(\alpha-\beta \rho^{2}\right) \rho}
$$


From the equality

$$
\varphi(v)=-\int_{-\infty}^{\tau_{1}} \psi(\mathcal{V}(\tau ; v)) \mathrm{d} \tau-\int_{\tau_{1}}^{0} \psi(\mathcal{V}(\tau ; v)) \mathrm{d} \tau=-\int_{\tau_{1}}^{0} \psi(\mathcal{V}(\tau ; v)) \mathrm{d} \tau
$$

we deduce that

$$
|\varphi(v)| \leq\left|\tau_{1}\right|\|\psi\|_{C^{0}} \leq \int_{r_{1}}^{r_{2}} \frac{\mathrm{d} \rho}{\left(\alpha-\beta \rho^{2}\right) \rho}\|\psi\|_{C^{0}} .
$$

Assume now that $r_{2} \leq|v|<r$. There is $\tau_{2} \geq 0$ such that $v=\mathcal{V}\left(\tau_{2} ; r_{2} \frac{v}{|v|}\right)$ and therefore

$$
\begin{aligned}
\varphi(v) & =-\int_{-\infty}^{0} \psi(\mathcal{V}(\tau ; v)) \mathrm{d} \tau=-\int_{-\infty}^{0} \psi\left(\mathcal{V}\left(\tau+\tau_{2} ; r_{2} \frac{v}{|v|}\right)\right) \mathrm{d} \tau \\
& =-\int_{-\infty}^{-\tau_{2}} \psi\left(\mathcal{V}\left(\tau+\tau_{2} ; r_{2} \frac{v}{|v|}\right)\right) \mathrm{d} \tau=-\int_{-\infty}^{0} \psi\left(\mathcal{V}\left(\tau ; r_{2} \frac{v}{|v|}\right)\right) \mathrm{d} \tau=\varphi\left(r_{2} \frac{v}{|v|}\right) .
\end{aligned}
$$

In particular, the restriction of $\varphi$ on $r_{2} \leq|v|<r$ satisfies the same bound as in (17)

$$
|\varphi(v)| \leq \int_{r_{1}}^{r_{2}} \frac{\mathrm{d} \rho}{\left(\alpha-\beta \rho^{2}\right) \rho}\|\psi\|_{C^{0}}, \quad r_{2} \leq|v|<r .
$$

It is easily seen that $\varphi$ is $C^{1}$ on $0 \leq|v|<r$. For that it is sufficient to consider $r_{1} \leq|v| \leq r_{2}$. Notice that

$$
\frac{\partial \mathcal{V}}{\partial v}(\tau ; v)=\frac{|\mathcal{V}(\tau ; v)|}{|v|}\left(I-\frac{\mathcal{V}(\tau ; v) \otimes \mathcal{V}(\tau ; v)}{r^{2}}\left(1-e^{-2 \alpha \tau}\right)\right)
$$

and therefore the gradient of $\varphi$ remains bounded on $r_{1} \leq|v| \leq r_{2}$

$$
\nabla_{v} \varphi(v)=-\int_{\tau_{1}}^{0} \frac{t}{\partial v}(\tau ; v) \nabla \psi(\mathcal{V}(\tau ; v)) \mathrm{d} \tau
$$

since on the interval $\tau \in\left[\tau_{1}, 0\right]$ we have $|\mathcal{V}(\tau ; v)| \in\left[r_{1},|v|\right] \subset\left[r_{1}, r_{2}\right]$. Taking now as definition for $|v|=r$

$$
\varphi(v)=\varphi\left(r_{2} \frac{v}{|v|}\right)
$$

we obtain a bounded $C^{1}$ function on $|v| \leq r$ satisfying

$$
-\left(\alpha-\beta|v|^{2}\right) v \cdot \nabla_{v} \varphi=\psi(v),|\varphi(v)| \leq \int_{r_{1}}^{r_{2}} \frac{\mathrm{d} \rho}{\left(\alpha-\beta \rho^{2}\right) \rho}\|\psi\|_{C^{0}},|v| \leq r .
$$

We proceed similarly in order to extend the above function for $|v|>r$. We have for any $s>0$

$$
-\varphi(\mathcal{V}(s ; v))+\varphi(v)=\int_{0}^{s} \psi(\mathcal{V}(\tau ; v)) \mathrm{d} \tau,|v|>r .
$$

As $\lim _{s \rightarrow+\infty} \mathcal{V}(s ; v)=r \frac{v}{|v|}$ we must take

$$
\varphi(v)=\lim _{s \rightarrow+\infty}\left\{\varphi(\mathcal{V}(s ; v))+\int_{0}^{s} \psi(\mathcal{V}(\tau ; v)) \mathrm{d} \tau\right\}=\varphi\left(r \frac{v}{|v|}\right)+\int_{0}^{+\infty} \psi(\mathcal{V}(\tau ; v)) \mathrm{d} \tau, \quad|v|>r .
$$

Clearly, for any $|v|>r$ the function $\tau \rightarrow|\mathcal{V}(\tau ; v)|$ is strictly decreasing. Therefore, for any $r<|v| \leq r_{3}$ we have

$$
\varphi(v)=\varphi\left(r \frac{v}{|v|}\right)=\varphi\left(r_{2} \frac{v}{|v|}\right)
$$


since $|\mathcal{V}(\tau ; v)| \leq|v| \leq r_{3}$ and $\psi(\mathcal{V}(\tau ; v))=0, \tau \geq 0$. If $r_{3}<|v|<r_{4}$ let us consider $\tau_{4}<0<\tau_{3}$ such that $\left|\mathcal{V}\left(\tau_{3} ; v\right)\right|=r_{3}<r_{4}=\left|\mathcal{V}\left(\tau_{4} ; v\right)\right|$. The time interval between $\tau_{4}$ and $\tau_{3}$ is given by

$$
\left|\tau_{3}\right|+\left|\tau_{4}\right|=\tau_{3}-\tau_{4}=\int_{r_{4}}^{r_{3}} \frac{\mathrm{d} \rho}{\left(\alpha-\beta \rho^{2}\right) \rho}<+\infty,
$$

and therefore one gets for $r_{3}<|v|<r_{4}$

$$
\begin{aligned}
|\varphi(v)| & \leq\left|\varphi\left(r \frac{v}{|v|}\right)\right|+\left|\int_{0}^{\tau_{3}} \psi(\mathcal{V}(\tau ; v)) \mathrm{d} \tau\right| \\
& \leq\left[\int_{r_{1}}^{r_{2}} \frac{\mathrm{d} \rho}{\left(\alpha-\beta \rho^{2}\right) \rho}+\int_{r_{4}}^{r_{3}} \frac{\mathrm{d} \rho}{\left(\alpha-\beta \rho^{2}\right) \rho}\right]\|\psi\|_{C^{0}} .
\end{aligned}
$$

Consider now $|v| \geq r_{4}$. There is $\tau_{4} \geq 0$ such that $r_{4} \frac{v}{v \mid}=\mathcal{V}\left(\tau_{4} ; v\right)$ implying that

$$
\begin{aligned}
\varphi(v) & =\varphi\left(r \frac{v}{|v|}\right)+\int_{0}^{+\infty} \psi(\mathcal{V}(\tau ; v)) \mathrm{d} \tau=\varphi\left(r \frac{v}{|v|}\right)+\int_{\tau_{4}}^{+\infty} \psi(\mathcal{V}(\tau ; v)) \mathrm{d} \tau \\
& =\varphi\left(r \frac{v}{|v|}\right)+\int_{0}^{+\infty} \psi\left(\mathcal{V}\left(\tau ; \mathcal{V}\left(\tau_{4} ; v\right)\right)\right) \mathrm{d} \tau=\varphi\left(r \frac{v}{|v|}\right)+\int_{0}^{+\infty} \psi\left(\mathcal{V}\left(\tau ; r_{4} \frac{v}{|v|}\right)\right) \mathrm{d} \tau \\
& =\varphi\left(r_{4} \frac{v}{|v|}\right) .
\end{aligned}
$$

We deduce that the restriction of $\varphi$ on $\left\{v:|v| \geq r_{4}\right\}$ satisfies the same bound as in (18). Moreover the function $\varphi$ is $C^{1}$ on $\{v:|v| \geq r\}$, with bounded derivatives. Indeed, it is sufficient to consider only the case $r_{3} \leq|v| \leq r_{4}$, observing that

$$
\begin{aligned}
& \nabla_{v} \varphi(v)=\frac{r_{2}}{|v|}\left(I-\frac{v \otimes v}{|v|^{2}}\right) \nabla_{v} \varphi\left(r_{2} \frac{v}{|v|}\right)+\int_{0}^{\tau_{3}} \frac{t}{\partial \mathcal{V}}(\tau ; v) \nabla \psi(\mathcal{V}(\tau ; v)) \mathrm{d} \tau \\
& |\mathcal{V}(\tau ; v)| \in\left[r_{3},|v|\right] \subset\left[r_{3}, r_{4}\right], \tau \in\left[0, \tau_{3}\right], \quad\left|\tau_{3}\right|+\left|\tau_{4}\right|=\int_{r_{4}}^{r_{3}} \frac{\mathrm{d} \rho}{\left(\alpha-\beta \rho^{2}\right) \rho}<+\infty .
\end{aligned}
$$

By construction we have $-\left(\alpha-\beta|v|^{2}\right) v \cdot \nabla_{v} \varphi=\psi(v),|v|>r$.

Consider a $C^{1}$ decreasing function on $\mathbb{R}_{+}$such that $\left.\chi\right|_{[0,1]}=1, \chi_{[2,+\infty[}=0$. We know that

$$
\int_{\mathbb{R}^{d}}\left(\alpha-\beta|v|^{2}\right) v \cdot \nabla_{v}\left\{\varphi(v) \chi\left(\frac{|v|}{R}\right)\right\} F(v) \mathrm{d} v=0, \quad R>0
$$

saying that

$$
\int_{\mathbb{R}^{d}} \chi\left(\frac{|v|}{R}\right)\left(\alpha-\beta|v|^{2}\right) v \cdot \nabla_{v} \varphi F(v) \mathrm{d} v+\int_{\mathbb{R}^{d}}\left(\alpha-\beta|v|^{2}\right) \varphi(v) \frac{|v|}{R} \chi^{\prime}\left(\frac{|v|}{R}\right) F(v) \mathrm{d} v=0 .
$$

Since $\varphi$ and $\psi=-\left(\alpha-\beta|v|^{2}\right) v \cdot \nabla_{v} \varphi$ are bounded and $F$ has finite mass and kinetic energy, we can pass to the limit for $R \rightarrow+\infty$, using the dominated convergence theorem. We obtain for any $C^{1}$ function $\psi$, with compact support in ${ }^{c}(\{0\} \cup r \mathbb{S})$

$$
\int_{\mathbb{R}^{d}} \psi(v) F(v) \mathrm{d} v=-\int_{\mathbb{R}^{d}}\left(\alpha-\beta|v|^{2}\right) v \cdot \nabla_{v} \varphi F(v) \mathrm{d} v=0
$$

Actually the previous equality holds true for any continuous function $\psi$ with compact support in ${ }^{c}(\{0\} \cup r \mathbb{S})$, since $\int_{\mathbb{R}^{d}} F(v) \mathrm{d} v<+\infty$, so that $\operatorname{supp} F \subset\{0\} \cup r \mathbb{S}$. 
In order to obtain stability for $\left(f^{\varepsilon_{k}}\right)_{k}$ we need to avoid the unstable equilibrium $v=0$. For that we assume that the initial support is away from zero speed: there is $r_{0}>0$ (eventually small, let us say $\left.r_{0}<r\right)$ such that

$$
\operatorname{supp} f^{\text {in }} \subset\left\{(x, v) \in \mathbb{R}^{d} \times \mathbb{R}^{d}:|v| \geq r_{0}\right\} .
$$

Proposition 3.1 Under the hypothesis (19) we have for any $\varepsilon>0$ small enough

$$
\operatorname{supp} f^{\varepsilon}(t) \subset\left\{(x, v) \in \mathbb{R}^{d} \times \mathbb{R}^{d}:|v| \geq r_{0}\right\}, \quad t \in \mathbb{R}_{+} .
$$

Proof. Take $\varepsilon>0$ such that $\varepsilon\|a\|_{L^{\infty}}<2 \alpha r /(3 \sqrt{3})$ and $\rho_{1}^{\varepsilon}\left(-\|a\|_{L^{\infty}}\right)<r_{0}$. For any continuous function $\psi=\psi(x, v)$ with compact support in $\mathbb{R}^{d} \times\left\{v:|v|<r_{0}\right\}$ we have

$$
\begin{aligned}
\int_{\mathbb{R}^{d} \times \mathbb{R}^{d}} \psi(x, v) f^{\varepsilon}(t, x, v) \mathrm{d}(x, v) & =\int_{\mathbb{R}^{d} \times \mathbb{R}^{d}} \psi\left(X^{\varepsilon}(t ; 0, x, v), V^{\varepsilon}(t ; 0, x, v)\right) f^{\mathrm{in}}(x, v) \mathrm{d}(x, v) \\
& =\int_{\mathbb{R}^{d} \times \mathbb{R}^{d}} \psi\left(X^{\varepsilon}(t ; 0, x, v), V^{\varepsilon}(t ; 0, x, v)\right) \mathbf{1}_{\left\{|v| \geq r_{0}\right\}} f^{\mathrm{in}}(x, v) \mathrm{d}(x, v) .
\end{aligned}
$$

But for any $|v| \geq r_{0}>\rho_{1}^{\varepsilon}$ we know by Proposition 2.7 that $\left|V^{\varepsilon}(t ; 0, x, v)\right|>|v| \geq r_{0}$, implying that $\psi\left(X^{\varepsilon}(t), V^{\varepsilon}(t)\right)=0$. Therefore one gets $\int_{\mathbb{R}^{d} \times \mathbb{R}^{d}} \psi(x, v) f^{\varepsilon}(t, x, v) \mathrm{d}(x, v)=0$ saying that $\operatorname{supp} f^{\varepsilon}(t) \subset\left\{(x, v):|v| \geq r_{0}\right\}$.

We are ready now to establish the model satisfied by the limit measure $f$. The idea is to use the weak formulation of (11), (12) with test functions which are constant along the flow of $\left(\alpha-\beta|v|^{2}\right) v \cdot \nabla_{v}$, in order to get rid of the term in $\frac{1}{\varepsilon}$. These functions are those depending on $x$ and $\frac{v}{|v|}$. Surely, the invariants $\frac{v}{|v|}$ have no continuous extensions in $v=0$, but we will see that we can use it, since our measures $f^{\varepsilon}$ vanish around $v=0$.

Proof. (of Theorem 1.1) We already know that $f$ satisfies (7). Actually, since supp $f^{\varepsilon}(t) \subset$ $\left\{(x, v):|v| \geq r_{0}\right\}, t \in \mathbb{R}_{+}, \varepsilon>0$, we deduce that $\operatorname{supp} f(t) \subset\left\{(x, v):|v| \geq r_{0}\right\}$ and finally supp $f(t) \subset \mathbb{R}^{d} \times r \mathbb{S}, t \in \mathbb{R}_{+}$. We have to establish (6) and find the initial data. Consider a $C^{1}$ decreasing function $\chi$ on $\mathbb{R}_{+}$such that $\left.\chi\right|_{[0,1]}=1, \chi_{[2,+\infty[}=0$. For any $\eta=\eta(t) \in C_{c}^{1}\left(\mathbb{R}_{+}\right)$, $\varphi=\varphi(x, v) \in C_{c}^{1}\left(\mathbb{R}^{d} \times \mathbb{R}^{d}\right)$ we construct the test function

$$
\theta(t, x, v)=\eta(t)\left[1-\chi\left(\frac{2|v|}{r_{0}}\right)\right] \varphi\left(x, r \frac{v}{|v|}\right) .
$$

Notice that $\theta$ is $C^{1}$ and $\theta=0$ for $|v| \leq \frac{r_{0}}{2}$. When applying the weak formulation of (11)-(12) with $\theta$, the term in $\frac{1}{\varepsilon}$ vanishes. Indeed, we can write

$$
\begin{aligned}
\frac{1}{\varepsilon} \int_{\mathbb{R}_{+}} \int_{\mathbb{R}^{d} \times \mathbb{R}^{d}} \eta(t) & \left(\alpha-\beta|v|^{2}\right) v \cdot \nabla_{v}\left\{\left[1-\chi\left(\frac{2|v|}{r_{0}}\right)\right] \varphi\left(x, r \frac{v}{|v|}\right)\right\} f^{\varepsilon}(t, x, v) \mathrm{d}(x, v) \mathrm{d} t \\
= & \frac{1}{\varepsilon} \int_{\mathbb{R}_{+}} \eta(t) \int_{|v| \geq r_{0}}\left(\alpha-\beta|v|^{2}\right) v \cdot \nabla_{v}\left\{\varphi\left(x, r \frac{v}{|v|}\right)\right\} f^{\varepsilon}(t, x, v) \mathrm{d}(x, v) \mathrm{d} t=0 .
\end{aligned}
$$


For the term containing $\partial_{t} \theta$ we obtain the following limit when $k \rightarrow+\infty$

$$
\begin{aligned}
T_{1}^{k}:=\int_{\mathbb{R}_{+}} \int_{\mathbb{R}^{d} \times \mathbb{R}^{d}} \partial_{t} \theta f^{\varepsilon_{k}}(t, x, v) \mathrm{d}(x, v) \mathrm{d} t & \rightarrow \int_{\mathbb{R}_{+}} \int_{\mathbb{R}^{d} \times \mathbb{R}^{d}} \partial_{t} \theta f(t, x, v) \mathrm{d}(x, v) \mathrm{d} t \\
& =\int_{\mathbb{R}_{+}} \eta^{\prime}(t) \int_{|v| \geq r_{0}} \varphi\left(x, r \frac{v}{|v|}\right) f(t, x, v) \mathrm{d}(x, v) \mathrm{d} t \\
& =\int_{\mathbb{R}_{+}} \eta^{\prime}(t) \int_{|v|=r} \varphi\left(x, r \frac{v}{|v|}\right) f(t, x, v) \mathrm{d}(x, v) \mathrm{d} t \\
& =\int_{\mathbb{R}_{+}} \eta^{\prime}(t) \int_{|v|=r} \varphi(x, v) f(t, x, v) \mathrm{d}(x, v) \mathrm{d} t \\
& =\int_{\mathbb{R}_{+}} \int_{\mathbb{R}^{d} \times \mathbb{R}^{d}} \partial_{t}(\eta \varphi) f(t, x, v) \mathrm{d}(x, v) \mathrm{d} t .
\end{aligned}
$$

Similarly, one gets

$$
\begin{aligned}
T_{2}^{k}:=\int_{\mathbb{R}_{+}} \int_{\mathbb{R}^{d} \times \mathbb{R}^{d}} v \cdot \nabla_{x} \theta f^{\varepsilon_{k}}(t, x, v) \mathrm{d}(x, v) \mathrm{d} t & \rightarrow \int_{\mathbb{R}_{+}} \int_{\mathbb{R}^{d} \times \mathbb{R}^{d}} v \cdot \nabla_{x} \theta f(t, x, v) \mathrm{d}(x, v) \mathrm{d} t \\
& =\int_{\mathbb{R}_{+}} \int_{\mathbb{R}^{d} \times \mathbb{R}^{d}} v \cdot \nabla_{x}(\eta \varphi) f(t, x, v) \mathrm{d}(x, v) \mathrm{d} t .
\end{aligned}
$$

For the term containing $a \cdot \nabla_{v} \theta$ notice that on the set $|v| \geq r_{0}$ we have

$$
a \cdot \nabla_{v} \theta=\eta(t) a \cdot \nabla_{v}\left\{\varphi\left(x, r \frac{v}{|v|}\right)\right\}=\eta(t) \frac{r}{|v|} a \cdot\left(I-\frac{v \otimes v}{|v|^{2}}\right)\left(\nabla_{v} \varphi\right)\left(x, r \frac{v}{|v|}\right)
$$

and therefore we obtain

$$
\begin{aligned}
T_{3}^{k}:=\int_{\mathbb{R}_{+}} \int_{\mathbb{R}^{d} \times \mathbb{R}^{d}} a \cdot \nabla_{v} \theta f^{\varepsilon_{k}}(t, x, v) \mathrm{d}(x, v) \mathrm{d} t \rightarrow \int_{\mathbb{R}_{+}} \int_{\mathbb{R}^{d} \times \mathbb{R}^{d}} a \cdot \nabla_{v} \theta f(t, x, v) \mathrm{d}(x, v) \mathrm{d} t \\
\quad=\int_{\mathbb{R}_{+}} \eta(t) \int_{|v| \geq r_{0}} \frac{r}{|v|}\left(I-\frac{v \otimes v}{|v|^{2}}\right) a \cdot\left(\nabla_{v} \varphi\right)\left(x, r \frac{v}{|v|}\right) f(t, x, v) \mathrm{d}(x, v) \mathrm{d} t \\
=\int_{\mathbb{R}_{+}} \int_{\mathbb{R}^{d} \times \mathbb{R}^{d}}\left(I-\frac{v \otimes v}{|v|^{2}}\right) a \cdot \nabla_{v}(\eta \varphi) f(t, x, v) \mathrm{d}(x, v) \mathrm{d} t .
\end{aligned}
$$

For treating the term involving the initial condition, we write

$$
\begin{aligned}
T_{4}:=\int_{\mathbb{R}^{d} \times \mathbb{R}^{d}} \theta(0, x, v) f^{\mathrm{in}}(x, v) \mathrm{d}(x, v) & =\int_{\mathbb{R}^{d} \times \mathbb{R}^{d}} \eta(0) \varphi\left(x, r \frac{v}{|v|}\right) f^{\text {in }}(x, v) \mathrm{d}(x, v) \\
& =\int_{\mathbb{R}^{d} \times \mathbb{R}^{d}} \eta(0) \varphi(x, v)\left\langle f^{\text {in }}\right\rangle(x, v) \mathrm{d}(x, v) .
\end{aligned}
$$

Passing to the limit for $k \rightarrow+\infty$ in the weak formulation $T_{1}^{k}+T_{2}^{k}+T_{3}^{k}+T_{4}=0$ yields the problem

$$
\partial_{t} f+\operatorname{div}_{x}\{f v\}+\operatorname{div}_{v}\left\{f\left(I-\frac{v \otimes v}{|v|^{2}}\right) a\right\}=0, \quad f(0)=\left\langle f^{\text {in }}\right\rangle
$$

as desired. 
Remark 3.1 The constraint (7) is propagated by the evolution equation (6). This comes by the fact that the flow $(X, V)$ associated to the field $v \cdot \nabla_{x}+\left(I-\frac{v \otimes v}{|v|^{2}}\right) a \cdot \nabla_{v}$ leaves invariant $\mathbb{R}^{d} \times r \mathbb{S}$. Indeed, if $(X, V)$ solves

$$
\begin{gathered}
\frac{\mathrm{d} X}{\mathrm{~d} s}=V(s), \frac{\mathrm{d} V}{\mathrm{~d} s}=\left(I-\frac{V(s) \otimes V(s)}{|V(s)|^{2}}\right) a(s, X(s)) \\
X(s ; 0, x, v)=x, \quad V(s ; 0, x, v)=v \neq 0
\end{gathered}
$$

then

$$
\frac{1}{2} \frac{\mathrm{d}}{\mathrm{d} s}|V(s)|^{2}=\left(I-\frac{V(s) \otimes V(s)}{|V(s)|^{2}}\right) a(s, X(s)) \cdot V(s)=0
$$

saying that $|V(s ; 0, x, v)|=|v|$ for any $(s, x) \in \mathbb{R}_{+} \times \mathbb{R}^{d}$. In particular, for any continuous function $\psi=\psi(x, v)$ with compact support in ${ }^{c}\left(\mathbb{R}^{d} \times r \mathbb{S}\right)$ we have

$$
\begin{aligned}
\int_{\mathbb{R}^{d} \times \mathbb{R}^{d}} \psi(x, v) f(s, x, v) \mathrm{d}(x, v) & =\int_{\mathbb{R}^{d} \times \mathbb{R}^{d}} \psi(X(s ; 0, x, v), V(s ; 0, x, v))\left\langle f^{\mathrm{in}}\right\rangle(x, v) \mathrm{d}(x, v) \\
& =\int_{|v|=r} \psi(X(s ; 0, x, v), V(s ; 0, x, v))\left\langle f^{\mathrm{in}}\right\rangle(x, v) \mathrm{d}(x, v)=0
\end{aligned}
$$

since $\operatorname{supp}\left\langle f^{\text {in }}\right\rangle \subset \mathbb{R}^{d} \times r \mathbb{S}$. Therefore for any $s \in \mathbb{R}_{+}$we have $\operatorname{supp} f(s) \subset \mathbb{R}^{d} \times r \mathbb{S}$ implying that $\operatorname{div}_{v}\left\{f(s)\left(\alpha-\beta|v|^{2}\right) v\right\}=0, s \in \mathbb{R}_{+}$.

Remark 3.2 By the uniqueness of the solution for (6) with initial data $\left\langle f^{\text {in }}\right\rangle$, we deduce that all the family $\left(f^{\varepsilon}\right)_{\varepsilon}$ converges weakly $\star$ in $L^{\infty}\left(\mathbb{R}_{+} ; \mathcal{M}_{b}\left(\mathbb{R}^{d} \times \mathbb{R}^{d}\right)\right)$.

\section{The non linear problem}

Up to now we considered the stability of the linear problems (11)-(12) for a given smooth field $a=a(t, x) \in L^{\infty}\left(\mathbb{R}_{+} ; W^{1, \infty}\left(\mathbb{R}^{d}\right)\right)$. We concentrate now on the non linear problem

$$
\partial_{t} f^{\varepsilon}+\operatorname{div}_{x}\left\{f^{\varepsilon} v\right\}+\operatorname{div}_{v}\left\{f^{\varepsilon} a^{\varepsilon}\right\}+\frac{1}{\varepsilon}\left\{f^{\varepsilon}\left(\alpha-\beta|v|^{2}\right) v\right\}=0, \quad(t, x, v) \in \mathbb{R}_{+} \times \mathbb{R}^{d} \times \mathbb{R}^{d}
$$

with $a^{\varepsilon}=-\nabla_{x} U \star \rho^{\varepsilon}-H \star f^{\varepsilon}$. The well posedness of the non linear equation (20) comes by fixed point arguments in suitable spaces of measures, and it has been discussed in [10, 4] in the measure solution framework. We summarize next the properties of the solutions $\left(f^{\varepsilon}\right)_{\varepsilon>0}$.

Proposition 4.1 Assume $h \in C_{b}^{1}\left(\mathbb{R}^{d}\right), U \in C_{b}^{2}\left(\mathbb{R}^{d}\right)$ and $\left(1+|v|^{2}\right) f^{\text {in }} \in \mathcal{M}_{b}^{+}\left(\mathbb{R}^{d} \times \mathbb{R}^{d}\right)$. For all $\varepsilon>0$, there is a unique solution $\left(f^{\varepsilon}, a^{\varepsilon}\right) \in C\left(\mathbb{R}_{+} ; \mathcal{P}_{1}\left(\mathbb{R}^{d} \times \mathbb{R}^{d}\right)\right) \times L^{\infty}\left(\mathbb{R}_{+} ; W^{1, \infty}\left(\mathbb{R}^{d}\right)\right)$ to

$$
\begin{gathered}
\partial_{t} f^{\varepsilon}+\operatorname{div}_{x}\left\{f^{\varepsilon} v\right\}+\operatorname{div}_{v}\left\{f^{\varepsilon} a^{\varepsilon}\right\}+\frac{1}{\varepsilon} \operatorname{div}_{v}\left\{f^{\varepsilon}\left(\alpha-\beta|v|^{2}\right) v\right\}=0, \quad(t, x, v) \in \mathbb{R}_{+} \times \mathbb{R}^{d} \times \mathbb{R}^{d} \\
a^{\varepsilon}=-\nabla_{x} U \star \int_{\mathbb{R}^{d}} f^{\varepsilon} \mathrm{d} v-H \star f^{\varepsilon}, \quad H(x, v)=h(x) v
\end{gathered}
$$


with initial data $f^{\varepsilon}(0)=f^{\text {in }}$, satisfying the uniform bounds

$$
\begin{gathered}
\sup _{\varepsilon>0, t \in \mathbb{R}_{+}} \int_{\mathbb{R}^{d} \times \mathbb{R}^{d}}|v|^{2} f^{\varepsilon}(t, x, v) \mathrm{d}(x, v)<+\infty \\
\sup _{\varepsilon>0}\left\|a^{\varepsilon}\right\|_{L^{\infty}\left(\mathbb{R}_{+} ; L^{\infty}\left(\mathbb{R}^{d}\right)\right)}=: A<+\infty, \sup _{\varepsilon>0}\left\|\nabla_{x} a^{\varepsilon}\right\|_{L^{\infty}\left(\mathbb{R}_{+} ; L^{\infty}\left(\mathbb{R}^{d}\right)\right)}=: A_{1}<+\infty .
\end{gathered}
$$

Moreover, if the initial condition satisfies

$$
\operatorname{supp} f^{\text {in }} \subset\left\{(x, v) \in \mathbb{R}^{d} \times \mathbb{R}^{d}:|x| \leq L_{0}, r_{0} \leq|v| \leq R_{0}\right\}
$$

for some $L_{0}>0,0<r_{0}<r<R_{0}<+\infty$, then for any $\varepsilon>0$ small enough we have

$$
\operatorname{supp} f^{\varepsilon}(t) \subset\left\{(x, v) \in \mathbb{R}^{d} \times \mathbb{R}^{d}:|x| \leq L_{0}+t R_{0}, r_{0} \leq|v| \leq R_{0}\right\}, \quad t \in \mathbb{R}_{+} .
$$

Proof. Here, we only justify the uniform bounds in $\varepsilon$, the rest is a direct application of the results in [10, 4]. The divergence form of (21) guarantees the mass conservation

$$
\int_{\mathbb{R}^{d} \times \mathbb{R}^{d}} f^{\varepsilon}(t, x, v) \mathrm{d}(x, v)=\int_{\mathbb{R}^{d} \times \mathbb{R}^{d}} f^{\mathrm{in}}(x, v) \mathrm{d}(x, v), \quad t \in \mathbb{R}_{+} .
$$

Notice that the term $-\operatorname{div}_{v}\left\{f^{\varepsilon} H \star f^{\varepsilon}\right\}$ balances the momentum

$$
\int_{\mathbb{R}^{2 d}} v \operatorname{div}_{v}\left\{f^{\varepsilon} H \star f^{\varepsilon}\right\} \mathrm{d}(x, v)=\int_{\mathbb{R}^{4 d}} h\left(x-x^{\prime}\right)\left(v^{\prime}-v\right) f^{\varepsilon}\left(t, x^{\prime}, v^{\prime}\right) f^{\varepsilon}(t, x, v) \mathrm{d}\left(x^{\prime}, v^{\prime}\right) \mathrm{d}(x, v)=0
$$

and decreases the kinetic energy

$$
\begin{aligned}
\int_{\mathbb{R}^{2 d}}|v|^{2} \operatorname{div}_{v}\left\{f^{\varepsilon} H \star f^{\varepsilon}\right\} \mathrm{d}(x, v) & =2 \int_{\mathbb{R}^{4 d}} h\left(x-x^{\prime}\right)\left(v^{\prime}-v\right) \cdot v f^{\varepsilon}\left(t, x^{\prime}, v^{\prime}\right) f^{\varepsilon}(t, x, v) \mathrm{d}\left(x^{\prime}, v^{\prime}\right) \mathrm{d}(x, v) \\
& =-\int_{\mathbb{R}^{4 d}} h\left(x-x^{\prime}\right)\left|v-v^{\prime}\right|^{2} f^{\varepsilon}\left(t, x^{\prime}, v^{\prime}\right) f^{\varepsilon}(t, x, v) \mathrm{d}\left(x^{\prime}, v^{\prime}\right) \mathrm{d}(x, v) .
\end{aligned}
$$

In particular, as $|v|^{2} f^{\text {in }} \in \mathcal{M}_{b}^{+}\left(\mathbb{R}^{d} \times \mathbb{R}^{d}\right)$, then the kinetic energy $\int_{\mathbb{R}^{2 d}}|v|^{2} f^{\varepsilon}(t, x, v) \mathrm{d}(x, v)$ remains bounded, uniformly in time $t \in \mathbb{R}_{+}$and $\varepsilon>0$. Indeed, using the continuity equation one gets

$$
\int_{\mathbb{R}^{d} \times \mathbb{R}^{d}} v \cdot\left(\nabla_{x} U \star \rho^{\varepsilon}\right) f^{\varepsilon}(t, x, v) \mathrm{d}(x, v)=\frac{1}{2} \frac{\mathrm{d}}{\mathrm{d} t} \int_{\mathbb{R}^{d}}\left(U \star \rho^{\varepsilon}(t)\right)(x) \rho^{\varepsilon}(t, x) \mathrm{d} x
$$

and after multiplying (21) by $\frac{|v|^{2}}{2}$ together with (22), we obtain

$$
\begin{gathered}
\frac{\mathrm{d}}{\mathrm{d} t} \int_{\mathbb{R}^{d} \times \mathbb{R}^{d}}\left(\frac{|v|^{2}}{2}+\frac{U \star \rho^{\varepsilon}}{2}\right) f^{\varepsilon}(t, x, v) \mathrm{d}(x, v)-\frac{1}{\varepsilon} \int_{\mathbb{R}^{d} \times \mathbb{R}^{d}}\left(\alpha|v|^{2}-\beta|v|^{4}\right) f^{\varepsilon}(t, x, v) \mathrm{d}(x, v) \\
=-\frac{1}{2} \int_{\mathbb{R}^{4 d}} h\left(x-x^{\prime}\right)\left|v-v^{\prime}\right|^{2} f^{\varepsilon}\left(t, x^{\prime}, v^{\prime}\right) f^{\varepsilon}(t, x, v) \mathrm{d}\left(x^{\prime}, v^{\prime}\right) \mathrm{d}(x, v) \leq 0
\end{gathered}
$$

Consider now $t^{\varepsilon}$ a maximum point on $[0, T], T>0$, of the total energy

$$
W^{\varepsilon}(t)=\int_{\mathbb{R}^{d} \times \mathbb{R}^{d}}\left(\frac{|v|^{2}}{2}+\frac{U \star \rho^{\varepsilon}}{2}\right) f^{\varepsilon}(t, x, v) \mathrm{d}(x, v), \quad t \in[0, T] .
$$


If $t^{\varepsilon}=0$ then it is easily seen that for any $t \in[0, T]$

$$
\int_{\mathbb{R}^{d} \times \mathbb{R}^{d}} \frac{|v|^{2}}{2} f^{\varepsilon}(t, x, v) \mathrm{d}(x, v) \leq \int_{\mathbb{R}^{d} \times \mathbb{R}^{d}} \frac{|v|^{2}}{2} f^{\mathrm{in}}(x, v) \mathrm{d}(x, v)+\|U\|_{L^{\infty}}\left(\int_{\mathbb{R}^{d} \times \mathbb{R}^{d}} f^{\text {in }} \mathrm{d}(x, v)\right)^{2} .
$$

If $\left.\left.t^{\varepsilon} \in\right] 0, T\right]$ then $\frac{\mathrm{d}}{\mathrm{d} t} W^{\varepsilon}\left(t^{\varepsilon}\right) \geq 0$ implying from (23) by moment interpolation in $v$ that

$$
\sup _{\varepsilon>0, T>0} \int_{\mathbb{R}^{d} \times \mathbb{R}^{d}}\left(1+|v|^{4}\right) f^{\varepsilon}\left(t^{\varepsilon}, x, v\right) \mathrm{d}(x, v)<+\infty
$$

and thus the inequality $W^{\varepsilon}(t) \leq W^{\varepsilon}\left(t^{\varepsilon}\right), t \in[0, T]$ yields

$$
\begin{aligned}
\sup _{\varepsilon>0, t \in[0, T]} \int_{\mathbb{R}^{d} \times \mathbb{R}^{d}} \frac{|v|^{2}}{2} f^{\varepsilon}(t, x, v) \mathrm{d}(x, v) \leq & \sup _{\varepsilon>0, T>0} \int_{\mathbb{R}^{d} \times \mathbb{R}^{d}} \frac{|v|^{2}}{2} f^{\varepsilon}\left(t^{\varepsilon}, x, v\right) \mathrm{d}(x, v) \\
& +\|U\|_{L^{\infty}}\left(\int_{\mathbb{R}^{d} \times \mathbb{R}^{d}} f^{\text {in }} \mathrm{d}(x, v)\right)^{2}<+\infty .
\end{aligned}
$$

Therefore the kinetic energy remains bounded on $[0, T]$, uniformly with respect to $\varepsilon>0$, and the bound does not depend on $T>0$. The uniform bounds for $a^{\varepsilon}$ come immediately by convolution with $\nabla_{x} U$ and $H$, thanks to the uniform estimate

$$
\sup _{\varepsilon>0, t \in \mathbb{R}_{+}} \int_{\mathbb{R}^{d} \times \mathbb{R}^{d}}|v| f^{\varepsilon}(t, x, v)<+\infty .
$$

We analyze the support of $\left(f^{\varepsilon}\right)_{\varepsilon>0}$. Take $\varepsilon>0$ small enough such that $\varepsilon A<2 \alpha r /(3 \sqrt{3})$ and $\rho_{1}^{\varepsilon}(-A)<r_{0}, \rho_{3}^{\varepsilon}(A)<R_{0}$. By Proposition 3.1 we already know that

$$
\operatorname{supp} f^{\varepsilon}(t) \subset\left\{(x, v) \in \mathbb{R}^{d} \times \mathbb{R}^{d}:|v| \geq r_{0}\right\}, \quad t \in \mathbb{R}_{+} .
$$

For any continuous function $\psi=\psi(x, v)$ with compact support in $\mathbb{R}^{d} \times\left\{v \in \mathbb{R}^{d}:|v|>R_{0}\right\}$ we have

$$
\begin{aligned}
\int_{\mathbb{R}^{d} \times \mathbb{R}^{d}} \psi(x, v) f^{\varepsilon}(t, x, v) \mathrm{d}(x, v) & =\int_{\mathbb{R}^{d} \times \mathbb{R}^{d}} \psi\left(X^{\varepsilon}(t), V^{\varepsilon}(t)\right) f^{\mathrm{in}}(x, v) \mathrm{d}(x, v) \\
& =\int_{\mathbb{R}^{d} \times \mathbb{R}^{d}} \psi\left(X^{\varepsilon}(t), V^{\varepsilon}(t)\right) \mathbf{1}_{\left\{r_{0} \leq|v| \leq R_{0}\right\}} f^{\mathrm{in}}(x, v) \mathrm{d}(x, v) .
\end{aligned}
$$

We distinguish several cases:

1. If $r_{0} \leq|v|<\rho_{2}^{\varepsilon}(-A)$ we deduce by Proposition 2.7 that $|v|<\left|V^{\varepsilon}(t ; 0, x, v)\right| \leq \rho_{3}^{\varepsilon}(A)<$ $R_{0}, t \in \mathbb{R}_{+}, \varepsilon>0$.

2. If $\rho_{2}^{\varepsilon}(-A) \leq|v| \leq \rho_{3}^{\varepsilon}(A)$ we obtain by Proposition 2.6 that $\rho_{2}^{\varepsilon}(-A) \leq\left|V^{\varepsilon}(t ; 0, x, v)\right| \leq$ $\rho_{3}^{\varepsilon}(A)<R_{0}, \quad t \in \mathbb{R}_{+}, \varepsilon>0$.

3. If $\rho_{3}^{\varepsilon}(A)<|v| \leq R_{0}$ one gets thanks to Proposition $2.7 \rho_{2}^{\varepsilon}(-A) \leq\left|V^{\varepsilon}(t ; 0, x, v)\right|<$ $|v| \leq R_{0}$.

In all cases $\left(X^{\varepsilon}, V^{\varepsilon}\right)(t ; 0, x, v)$ remains outside the support of $\psi$, implying that

$$
\int_{\mathbb{R}^{d} \times \mathbb{R}^{d}} \psi(x, v) f^{\varepsilon}(t, x, v) \mathrm{d}(x, v)=0 .
$$


Thus for any $t \in \mathbb{R}_{+}$and $\varepsilon>0$ small enough one gets

$$
\operatorname{supp} f^{\varepsilon}(t) \subset\left\{(x, v) \in \mathbb{R}^{d} \times \mathbb{R}^{d}: r_{0} \leq|v| \leq R_{0}\right\}
$$

Consider $\theta \in C^{1}(\mathbb{R})$ non decreasing, verifying $\theta(u)=0$ if $u \leq 0, \theta(u)>0$ if $u>0$. Applying the weak formulation of (21) $-(22)$ with the test function $\theta\left(|x|-L_{0}-t R_{0}\right)$ yields

$$
\begin{aligned}
& \int_{\mathbb{R}^{d} \times \mathbb{R}^{d}} \theta\left(|x|-L_{0}-\right.\left.t R_{0}\right) f^{\varepsilon}(t, x, v) \mathrm{d}(x, v)=\int_{\mathbb{R}^{d} \times \mathbb{R}^{d}} \theta\left(|x|-L_{0}\right) f^{\mathrm{in}}(x, v) \mathrm{d}(x, v) \\
&+\int_{0}^{t} \int_{\mathbb{R}^{d} \times \mathbb{R}^{d}} \theta^{\prime}\left(|x|-L_{0}-s R_{0}\right)\left(v \cdot \frac{x}{|x|}-R_{0}\right) f^{\varepsilon}(s, x, v) \mathrm{d}(x, v) \mathrm{d} s \leq 0
\end{aligned}
$$

implying that supp $f^{\varepsilon}(t) \subset\left\{(x, v) \in \mathbb{R}^{d} \times \mathbb{R}^{d}:|x| \leq L_{0}+t R_{0}\right\}, t \in \mathbb{R}_{+}$.

The uniform bound for the total mass allows us to extract a sequence $\left(\varepsilon_{k}\right)_{k} \subset \mathbb{R}_{+}^{\star}$ convergent to 0 such that $\left(f^{\varepsilon_{k}}\right)_{k}$ converges weakly $\star$ in $L^{\infty}\left(\mathbb{R}_{+} ; \mathcal{M}_{b}\left(\mathbb{R}^{d} \times \mathbb{R}^{d}\right)\right)$. The treatment of the non linear term requires a little bit more, that is convergence in $C\left(\mathbb{R}_{+} ; \mathcal{P}_{1}\left(\mathbb{R}^{d} \times \mathbb{R}^{d}\right)\right)$ or at least in $C\left(\left[\delta,+\infty\left[; \mathcal{P}_{1}\left(\mathbb{R}^{d} \times \mathbb{R}^{d}\right)\right)\right.\right.$ for any $\delta>0$. The key argument for establishing that is emphasized by the lemma

Lemma 4.1 Consider $\varepsilon>0$ small enough.

1. For any $(x, v) \in \mathbb{R}^{d} \times \mathbb{R}^{d}$ with $r_{0} \leq|v|<\rho_{2}^{\varepsilon}(-A)-\varepsilon$, the first time $t_{1}^{\varepsilon}=t_{1}^{\varepsilon}(x, v)$ such that $\left|V^{\varepsilon}\left(t_{1}^{\varepsilon} ; 0, x, v\right)\right|=\rho_{2}^{\varepsilon}(-A)-\varepsilon$ satisfies

$$
t_{1}^{\varepsilon} \leq \frac{\varepsilon}{2 \beta r_{0}^{2}} \ln \left(\frac{r-r_{0}}{\varepsilon}\right) .
$$

2. For any $(x, v) \in \mathbb{R}^{d} \times \mathbb{R}^{d}$ with $\rho_{3}^{\varepsilon}(A)+\varepsilon<|v| \leq R_{0}$, the first time $t_{2}^{\varepsilon}=t_{2}^{\varepsilon}(x, v)$ such that $\left|V^{\varepsilon}\left(t_{2}^{\varepsilon} ; 0, x, v\right)\right|=\rho_{3}^{\varepsilon}(A)+\varepsilon$ satisfies

$$
t_{2}^{\varepsilon} \leq \frac{\varepsilon}{2 \beta r^{2}} \ln \left(\frac{R_{0}-r}{\varepsilon}\right)
$$

Proof. 1. During the time $\left[0, t_{1}^{\varepsilon}\right]$ the velocity modulus $\left|V^{\varepsilon}(t)\right|$ remains in $\left[r_{0}, \rho_{2}^{\varepsilon}(-A)-\varepsilon\right] \subset$ $\left[\rho_{1}^{\varepsilon}(-A), \rho_{2}^{\varepsilon}(-A)\right]$ and we can write for any $t \in\left[0, t_{1}^{\varepsilon}\right]$

$$
\frac{\varepsilon \frac{\mathrm{d}\left|V^{\varepsilon}\right|}{\mathrm{d} t}}{-\varepsilon A+\left(\alpha-\beta\left|V^{\varepsilon}(t)\right|^{2}\right)\left|V^{\varepsilon}(t)\right|} \geq \frac{\frac{\mathrm{d}\left|V^{\varepsilon}\right|}{\mathrm{d} t}}{a^{\varepsilon}\left(t, X^{\varepsilon}(t)\right) \cdot \frac{V^{\varepsilon}(t)}{\left|V^{\varepsilon}(t)\right|}+\frac{1}{\varepsilon}\left(\alpha-\beta\left|V^{\varepsilon}(t)\right|^{2}\right)\left|V^{\varepsilon}(t)\right|}=1
$$

since $-\varepsilon A+\left(\alpha-\beta u^{2}\right) u$ is positive for $u \in\left[\rho_{1}^{\varepsilon}(-A), \rho_{2}^{\varepsilon}(-A)\right]$. Integrating with respect to $t \in\left[0, t_{1}^{\varepsilon}\right]$ yields

$$
t_{1}^{\varepsilon}(x, v) \leq \varepsilon \int_{|v|}^{\rho_{2}^{\varepsilon}(-A)-\varepsilon} \frac{\mathrm{d} u}{-\varepsilon A+\left(\alpha-\beta u^{2}\right) u} \leq \varepsilon \int_{r_{0}}^{\rho_{2}^{\varepsilon}(-A)-\varepsilon} \frac{\mathrm{d} u}{-\varepsilon A+\left(\alpha-\beta u^{2}\right) u} .
$$


Recall that $\rho_{2}^{\varepsilon}(-A)$ is one of the roots of $u \rightarrow-\varepsilon A+\left(\alpha-\beta u^{2}\right) u$ and therefore a direct computation lead to

$-\varepsilon A+\left(\alpha-\beta u^{2}\right) u=\beta\left(\rho_{2}^{\varepsilon}-u\right)\left[u^{2}+u \rho_{2}^{\varepsilon}+\left(\rho_{2}^{\varepsilon}\right)^{2}-r^{2}\right] \geq 2 \beta r_{0}^{2}\left(\rho_{2}^{\varepsilon}-u\right), \quad u \in\left[r_{0}, \rho_{2}^{\varepsilon}\right], \varepsilon$ small enough implying that

$$
t_{1}^{\varepsilon}(x, v) \leq \frac{\varepsilon}{2 \beta r_{0}^{2}} \int_{r_{0}}^{\rho_{2}^{\varepsilon}-\varepsilon} \frac{\mathrm{d} u}{\rho_{2}^{\varepsilon}-u}=\frac{\varepsilon}{2 \beta r_{0}^{2}} \ln \left(\frac{\rho_{2}^{\varepsilon}-r_{0}}{\varepsilon}\right) \leq \frac{\varepsilon}{2 \beta r_{0}^{2}} \ln \left(\frac{r-r_{0}}{\varepsilon}\right) .
$$

2. During the time $\left[0, t_{2}^{\varepsilon}\right]$ the velocity modulus $\left|V^{\varepsilon}(t)\right|$ remains in $\left[\rho_{3}^{\varepsilon}(A)+\varepsilon, R_{0}\right] \subset\left[\rho_{3}^{\varepsilon}(A),+\infty[\right.$ and we can write for any $t \in\left[0, t_{2}^{\varepsilon}\right]$

$$
\frac{\varepsilon \frac{\mathrm{d}\left|V^{\varepsilon}\right|}{\mathrm{d} t}}{\varepsilon A+\left(\alpha-\beta\left|V^{\varepsilon}(t)\right|^{2}\right)\left|V^{\varepsilon}(t)\right|} \geq \frac{\frac{\mathrm{d}\left|V^{\varepsilon}\right|}{\mathrm{d} t}}{a^{\varepsilon}\left(t, X^{\varepsilon}(t)\right) \cdot \frac{V^{\varepsilon}(t)}{\left|V^{\varepsilon}(t)\right|}+\frac{1}{\varepsilon}\left(\alpha-\beta\left|V^{\varepsilon}(t)\right|^{2}\right)\left|V^{\varepsilon}(t)\right|}=1
$$

since $\varepsilon A+\left(\alpha-\beta u^{2}\right) u$ is negative for $u \in\left[\rho_{3}^{\varepsilon}(A),+\infty\left[\right.\right.$. Integrating with respect to $t \in\left[0, t_{2}^{\varepsilon}\right]$ yields

$$
t_{2}^{\varepsilon}(x, v) \leq \varepsilon \int_{|v|}^{\rho_{3}^{\varepsilon}(A)+\varepsilon} \frac{\mathrm{d} u}{\varepsilon A+\left(\alpha-\beta u^{2}\right) u} \leq \varepsilon \int_{R_{0}}^{\rho_{3}^{\varepsilon}(A)+\varepsilon} \frac{\mathrm{d} u}{\varepsilon A+\left(\alpha-\beta u^{2}\right) u} .
$$

By direct computation we obtain

$\varepsilon A+\left(\alpha-\beta u^{2}\right) u=-\beta\left(u-\rho_{3}^{\varepsilon}\right)\left[u^{2}+u \rho_{3}^{\varepsilon}+\left(\rho_{3}^{\varepsilon}\right)^{2}-r^{2}\right] \leq-2 \beta r^{2}\left(u-\rho_{3}^{\varepsilon}\right), \quad u \geq \rho_{3}^{\varepsilon}, \varepsilon$ small enough implying that

$$
t_{2}^{\varepsilon}(x, v) \leq \frac{\varepsilon}{2 \beta r^{2}} \int_{\rho_{3}^{\varepsilon}+\varepsilon}^{R_{0}} \frac{\mathrm{d} u}{u-\rho_{3}^{\varepsilon}}=\frac{\varepsilon}{2 \beta r^{2}} \ln \left(\frac{R_{0}-\rho_{3}^{\varepsilon}}{\varepsilon}\right) \leq \frac{\varepsilon}{2 \beta r^{2}} \ln \left(\frac{R_{0}-r}{\varepsilon}\right) .
$$

We intend to apply Arzela-Ascoli theorem in $C\left(\mathbb{R}_{+} ; \mathcal{P}_{1}\left(\mathbb{R}^{d} \times \mathbb{R}^{d}\right)\right)$ in order to extract a convergent sequence $\left(f^{\varepsilon_{k}}\right)_{k}$ with $\lim _{k \rightarrow+\infty} \varepsilon_{k}=0$. We need to establish the uniform equicontinuity of the family $\left(f^{\varepsilon}\right)_{\varepsilon>0}$. The argument below is essentially similar to arguments in [10].

Proposition 4.2 1. If the initial data is well prepared i.e., supp $f^{\text {in }} \subset\left\{(x, v) \in \mathbb{R}^{d} \times \mathbb{R}^{d}\right.$ : $\left.|x| \leq L_{0},|v|=r\right\}$ then there is a constant $C$ (not depending on $t \in \mathbb{R}_{+}, \varepsilon>0$ ) such that

$$
W_{1}\left(f^{\varepsilon}(t), f^{\varepsilon}(s)\right) \leq C|t-s|, \quad t, s \in \mathbb{R}_{+}, \varepsilon>0 .
$$

2. If supp $f^{\text {in }} \subset\left\{(x, v) \in \mathbb{R}^{d} \times \mathbb{R}^{d}:|x| \leq L_{0}, r_{0} \leq|v| \leq R_{0}\right\}$ then there is a constant $C$ (not depending on $t \in \mathbb{R}_{+}, \varepsilon>0$ ) such that for any $\delta>0$ we can find $\varepsilon_{\delta}$ satisfying

$$
W_{1}\left(f^{\varepsilon}(t), f^{\varepsilon}(s)\right) \leq C|t-s|, \quad t, s \geq \delta, \quad 0<\varepsilon<\varepsilon_{\delta} .
$$


Proof. 1. Consider $\varphi=\varphi(x, v)$ a Lipschitz function on $\mathbb{R}^{d} \times \mathbb{R}^{d}$ with $\operatorname{Lip}(\varphi) \leq 1$. For any $t, s \in \mathbb{R}_{+}, \varepsilon>0$ we have

$$
\begin{aligned}
\left|\int_{\mathbb{R}^{d} \times \mathbb{R}^{d}} \varphi\left(f^{\varepsilon}(t)-f^{\varepsilon}(s)\right) \mathrm{d}(x, v)\right| & =\left|\int_{\mathbb{R}^{d} \times \mathbb{R}^{d}}\left\{\varphi\left(X^{\varepsilon}(t), V^{\varepsilon}(t)\right)-\varphi\left(X^{\varepsilon}(s), V^{\varepsilon}(s)\right)\right\} f^{\text {in }}(x, v) \mathrm{d}(x, v)\right| \\
& \leq \int_{\mathbb{R}^{d} \times \mathbb{R}^{d}}\left\{\left|X^{\varepsilon}(t)-X^{\varepsilon}(s)\right|+\left|V^{\varepsilon}(t)-V^{\varepsilon}(s)\right|\right\} \mathbf{1}_{\{|v|=r\}} f^{\text {in } \mathrm{d}(x, v) .}
\end{aligned}
$$

Thanks to Proposition 2.6 we have for any $(\tau, x, v) \in \mathbb{R}_{+} \times \mathbb{R}^{d} \times r \mathbb{S}$

$$
\frac{\rho_{2}^{\varepsilon}(-A)-r}{\varepsilon} \leq \frac{\left|V^{\varepsilon}(\tau ; 0, x, v)\right|-r}{\varepsilon} \leq \frac{\rho_{3}^{\varepsilon}(A)-r}{\varepsilon}
$$

and it is easily seen, integrating the system of characteristics between $s$ and $t$, that

$$
\left|X^{\varepsilon}(t ; 0, x, v)-X^{\varepsilon}(s ; 0, x, v)\right|=\left|\int_{s}^{t} V^{\varepsilon}(\tau ; 0, x, v) \mathrm{d} \tau\right| \leq R_{0}|t-s|
$$

and

$$
\begin{aligned}
\left|V^{\varepsilon}(t ; 0, x, v)-V^{\varepsilon}(s ; 0, x, v)\right| & \leq\left|\int_{s}^{t}\left\{\left|a^{\varepsilon}\left(\tau, X^{\varepsilon}(\tau)\right)\right|+\frac{\left.|\alpha-\beta| V^{\varepsilon}(\tau)\right|^{2}|| V^{\varepsilon}(\tau) \mid}{\varepsilon}\right\} \mathrm{d} \tau\right| \\
& \leq|t-s|\left\{A+\beta\left(r+R_{0}\right) R_{0} \max \left(\frac{\rho_{3}^{\varepsilon}(A)-r}{\varepsilon}, \frac{r-\rho_{2}^{\varepsilon}(-A)}{\varepsilon}\right)\right\} .
\end{aligned}
$$

Our conclusion comes immediately by Propositions 2.3, 2.4.

2. Consider $\delta>0$ and $\varepsilon_{\delta}$ small enough such that $\frac{\varepsilon}{2 \beta r_{0}^{2}} \ln \left(\frac{r-r_{0}}{\varepsilon}\right)<\delta, \frac{\varepsilon}{2 \beta r^{2}} \ln \left(\frac{R_{0}-r}{\varepsilon}\right)<\delta$ for $0<\varepsilon<\varepsilon_{\delta}$. For any Lipschitz function $\varphi$ with $\operatorname{Lip}(\varphi) \leq 1$ and any $t, s \geq \delta$ we have

$$
\left|\int_{\mathbb{R}^{d} \times \mathbb{R}^{d}} \varphi\left(f^{\varepsilon}(t)-f^{\varepsilon}(s)\right) \mathrm{d}(x, v)\right| \leq \int_{\mathbb{R}^{d} \times \mathbb{R}^{d}}\left\{\left|X^{\varepsilon}(t)-X^{\varepsilon}(s)\right|+\left|V^{\varepsilon}(t)-V^{\varepsilon}(s)\right|\right\} \mathbf{1}_{\left\{r_{0} \leq|v| \leq R_{0}\right\}} f^{\text {in }} \mathrm{d}(x, v) .
$$

For any $(\tau, x) \in \mathbb{R}_{+} \times \mathbb{R}^{d}, \rho_{2}^{\varepsilon}(-A)-\varepsilon \leq|v| \leq \rho_{3}^{\varepsilon}(A)+\varepsilon$ we have by Propositions [2.6, 2.7

$$
\rho_{2}^{\varepsilon}(-A)-\varepsilon \leq\left|V^{\varepsilon}(\tau ; 0, x, v)\right| \leq \rho_{3}^{\varepsilon}(A)+\varepsilon
$$

The same conclusion holds true for any $\tau \geq \delta, x \in \mathbb{R}^{d}$ and $|v| \in\left[r_{0}, \rho_{2}^{\varepsilon}(-A)-\varepsilon[\cup] \rho_{3}^{\varepsilon}(A)+\varepsilon, R_{0}\right]$, thanks to Lemma 4.1, since $\delta>\max \left\{t_{1}^{\varepsilon}(x, v), t_{2}^{\varepsilon}(x, v)\right\}$ (after a time $\delta$, the velocity modulus $\left|V^{\varepsilon}(\tau ; 0, x, v)\right|$ is already in the set $\left.\left\{w: \rho_{2}^{\varepsilon}(-A)-\varepsilon<|w|<\rho_{3}^{\varepsilon}(A)+\varepsilon\right\}\right)$. Our statement follows as before, integrating the system of characteristics between $s$ and $t$.

Applying Arzela-Ascoli theorem, we deduce that there is a sequence $\left(\varepsilon_{k}\right)_{k} \subset \mathbb{R}_{+}^{\star}$, convergent to 0 such that

$$
\lim _{k \rightarrow+\infty} W_{1}\left(f^{\varepsilon_{k}}(t), f(t)\right)=0 \text { uniformly for } t \in[0, T], \quad T>0
$$

for some $f \in C\left(\mathbb{R}_{+} ; \mathcal{P}_{1}\left(\mathbb{R}^{d} \times \mathbb{R}^{d}\right)\right)$ if supp $f^{\text {in }} \subset\left\{(x, v) \in \mathbb{R}^{d} \times \mathbb{R}^{d}:|x| \leq L_{0},|v|=r\right\}$ and

$$
\lim _{k \rightarrow+\infty} W_{1}\left(f^{\varepsilon_{k}}(t), f(t)\right)=0 \text { uniformly for } t \in[\delta, T], \quad T>\delta>0
$$


for some $f \in C\left(\mathbb{R}_{+}^{\star} ; \mathcal{P}_{1}\left(\mathbb{R}^{d} \times \mathbb{R}^{d}\right)\right)$ if $\operatorname{supp} f^{\text {in }} \subset\left\{(x, v) \in \mathbb{R}^{d} \times \mathbb{R}^{d}:|x| \leq L_{0}, r_{0} \leq|v| \leq R_{0}\right\}$. It is easily seen that if the initial condition is well prepared then there is a constant $C$ cf. Proposition 4.2 such that $W_{1}(f(t), f(s)) \leq C|t-s|, \quad t, s \in \mathbb{R}_{+}$. The same is true for not prepared initial conditions $f^{\text {in }}$. Take $\delta>0$ and $\varepsilon_{\delta}$ as in Proposition 4.2. For any $0<\varepsilon<\varepsilon_{\delta}$ we have $W_{1}\left(f^{\varepsilon}(t), f^{\varepsilon}(s)\right) \leq C|t-s|, \quad t, s \geq \delta$. For $k$ large enough we have $\varepsilon_{k}<\varepsilon_{\delta}$ and therefore $W_{1}\left(f^{\varepsilon_{k}}(t), f^{\varepsilon_{k}}(s)\right) \leq C|t-s|, \quad t, s \geq \delta$. Passing to the limit as $k$ goes to infinity yields $W_{1}(f(t), f(s)) \leq C|t-s|, \quad t, s \geq \delta$. Since the constant $C$ does not depend on $\delta$ one gets

$$
W_{1}(f(t), f(s)) \leq C|t-s|, \quad t, s>0 .
$$

In particular we deduce that $f$ has a limit as $t$ goes to 0 since $\left(\mathcal{P}_{1}\left(\mathbb{R}^{d} \times \mathbb{R}^{d}\right), W_{1}\right)$ is a complete metric space and therefore we can extend $f$ by continuity at $t=0$. The extended function, still denoted by $f$, belongs to $C\left(\mathbb{R}_{+} ; \mathcal{P}_{1}\left(\mathbb{R}^{d} \times \mathbb{R}^{d}\right)\right)$ and satisfies

$$
W_{1}(f(t), f(s)) \leq C|t-s|, \quad t, s \in \mathbb{R}_{+} .
$$

The above convergence allows us to handle the non linear terms. We use the following standard argument [24, 10].

Lemma 4.2 Consider $f, g \in \mathcal{P}_{1}\left(\mathbb{R}^{d} \times \mathbb{R}^{d}\right)$ compactly supported supp $f \cup \operatorname{supp} g \subset\{(x, v) \in$ $\left.\mathbb{R}^{d} \times \mathbb{R}^{d}:|x| \leq L,|v| \leq R\right\}$, and let us consider

$$
a_{f}=-\nabla_{x} U \star \int_{\mathbb{R}^{d}} f \mathrm{~d} v-H \star f, \quad a_{g}=-\nabla_{x} U \star \int_{\mathbb{R}^{d}} g \mathrm{~d} v-H \star g .
$$

Then we have

$$
\left\|a_{f}-a_{g}\right\|_{L^{\infty}\left(\mathbb{R}^{3} \times B_{R}\right)} \leq\left\{\left\|\nabla_{x}^{2} U\right\|_{L^{\infty}}+\left(\|h\|_{L^{\infty}}^{2}+4 R^{2}\left\|\nabla_{x} h\right\|_{L^{\infty}}^{2}\right)^{1 / 2}\right\} W_{1}(f, g)
$$

where $B_{R}$ stands for the closed ball in $\mathbb{R}^{d}$ of center 0 and radius $R$.

Proof. Take $\pi$ to be a optimal transportation plan between $f$ and $g$. Then for any $x \in \mathbb{R}^{d}$ we have, using the marginals of $\pi$

$$
\begin{aligned}
\left|\left(\nabla_{x} U \star f\right)(x)-\left(\nabla_{x} U \star g\right)(x)\right| & =\left|\int_{\mathbb{R}^{d} \times \mathbb{R}^{d}} \nabla_{x} U\left(x-x^{\prime}\right)\left\{f\left(x^{\prime}, v^{\prime}\right)-g\left(x^{\prime}, v^{\prime}\right)\right\} \mathrm{d}\left(x^{\prime}, v^{\prime}\right)\right| \\
& =\left|\int_{\mathbb{R}^{d} \times \mathbb{R}^{d}} \int_{\mathbb{R}^{d} \times \mathbb{R}^{d}}\left[\nabla_{x} U\left(x-x^{\prime}\right)-\nabla_{x} U\left(x-x^{\prime \prime}\right)\right] \mathrm{d} \pi\left(x^{\prime}, v^{\prime}, x^{\prime \prime}, v^{\prime \prime}\right)\right| \\
& \leq\left\|\nabla_{x}^{2} U\right\|_{L^{\infty}} \int_{\mathbb{R}^{d} \times \mathbb{R}^{d}} \int_{\mathbb{R}^{d} \times \mathbb{R}^{d}}\left|x^{\prime}-x^{\prime \prime}\right| \mathrm{d} \pi\left(x^{\prime}, v^{\prime}, x^{\prime \prime}, v^{\prime \prime}\right) \\
& \leq\left\|\nabla_{x}^{2} U\right\|_{L^{\infty}} W_{1}(f, g) .
\end{aligned}
$$


The estimate for $H \star f-H \star g$ follows similarly observing that on the support of $\pi$, which is included in $\left\{\left(x^{\prime}, v^{\prime}, x^{\prime \prime}, v^{\prime \prime}\right) \in \mathbb{R}^{4 d}:\left|v^{\prime}\right| \leq R,\left|v^{\prime \prime}\right| \leq R\right\}$ we have

$$
\begin{aligned}
\mid h\left(x-x^{\prime}\right)\left(v-v^{\prime}\right)-h\left(x-x^{\prime \prime}\right) & \left(v-v^{\prime \prime}\right) \mid \\
& \leq\left|h\left(x-x^{\prime}\right)\left(v^{\prime \prime}-v^{\prime}\right)\right|+\left|h\left(x-x^{\prime}\right)-h\left(x-x^{\prime \prime}\right)\right|\left|v-v^{\prime \prime}\right| \\
& \leq\left(\|h\|_{L^{\infty}}^{2}+4 R^{2}\left\|\nabla_{x} h\right\|_{L^{\infty}}^{2}\right)^{1 / 2}\left(\left|x^{\prime}-x^{\prime \prime}\right|^{2}+\left|v^{\prime}-v^{\prime \prime}\right|^{2}\right)^{1 / 2} .
\end{aligned}
$$

We are ready now to prove Theorem 1.2 ,

Proof. (of Theorem 1.2) The arguments are the same as those in the proof of Theorem 1.1 except for the treatment of the non linear terms. We only concentrate on it. Consider $\left(f^{\varepsilon_{k}}\right)_{k}$ with $\lim _{k \rightarrow+\infty} \varepsilon_{k}=0$ such that $\lim _{k \rightarrow+\infty} W_{1}\left(f^{\varepsilon_{k}}(t), f(t)\right)=0$ uniformly for $t \in[0, T], T>0$ if supp $f^{\text {in }} \subset\left\{(x, v):|x| \leq L_{0},|v|=r\right\}$ and $\lim _{k \rightarrow+\infty} W_{1}\left(f^{\varepsilon_{k}}(t), f(t)\right)=0$ uniformly for $t \in[\delta, T], T>\delta>0$ if $\operatorname{supp} f^{\text {in }} \subset\left\{(x, v):|x| \leq L_{0}, r_{0} \leq|v| \leq R_{0}\right\}$ for some function $f \in C\left(\mathbb{R}_{+} ; \mathcal{P}_{1}\left(\mathbb{R}^{d} \times \mathbb{R}^{d}\right)\right)$. Thanks to Proposition 1.1 we deduce (for both prepared or not initial data) that

$$
\operatorname{supp} f(t) \subset\left\{(x, v) \in \mathbb{R}^{d} \times \mathbb{R}^{d}:|v|=r\right\}, \quad t>0 .
$$

The previous statement holds also true at $t=0$, by the continuity of $f$. The time evolution for the limit $f$ comes by using the particular test functions

$$
\theta(t, x, v)=\eta(t)\left[1-\chi\left(\frac{2|v|}{r_{0}}\right)\right] \varphi\left(x, r \frac{v}{|v|}\right)
$$

with $\eta \in C_{c}^{1}\left(\mathbb{R}_{+}\right), \varphi \in C_{c}^{1}\left(\mathbb{R}^{d} \times \mathbb{R}^{d}\right)$. From now on we consider only the not prepared initial data case (the other case is simpler). We recall the notation $a^{\varepsilon}=-\nabla_{x} U \star \int_{\mathbb{R}^{d}} f^{\varepsilon} \mathrm{d} v-H \star f^{\varepsilon}$ and we introduce $a=-\nabla_{x} U \star \int_{\mathbb{R}^{d}} f \mathrm{~d} v-H \star f$. Since $f$ satisfies the same bounds as $\left(f^{\varepsilon}\right)_{\varepsilon}$, we deduce that $\|a\|_{L^{\infty}} \leq A,\left\|\nabla_{x} a\right\|_{L^{\infty}} \leq A_{1}$. For any $\delta>0$ we can write

$$
\begin{aligned}
& \quad\left|\int_{\mathbb{R}_{+}} \int_{\mathbb{R}^{d} \times \mathbb{R}^{d}}\left\{a^{\varepsilon_{k}} \cdot \nabla_{v} \theta f^{\varepsilon_{k}}-a \cdot \nabla_{v} \theta f\right\} \mathrm{d}(x, v) \mathrm{d} t\right| \leq\left|\int_{0}^{\delta} \int_{\mathbb{R}^{d} \times \mathbb{R}^{d}} a^{\varepsilon_{k}} \cdot \nabla_{v} \theta f^{\varepsilon_{k}} \mathrm{~d}(x, v) \mathrm{d} t\right| \\
& \quad+\left|\int_{0}^{\delta} \int_{\mathbb{R}^{d} \times \mathbb{R}^{d}} a \cdot \nabla_{v} \theta f \mathrm{~d}(x, v) \mathrm{d} t\right|+\left|\int_{\delta}^{+\infty} \int_{\mathbb{R}^{d} \times \mathbb{R}^{d}}\left\{a^{\varepsilon_{k}} \cdot \nabla_{v} \theta f^{\varepsilon_{k}}-a \cdot \nabla_{v} \theta f\right\} \mathrm{d}(x, v) \mathrm{d} t\right| \\
& \leq 2 A \delta\left\|\nabla_{v} \theta\right\|_{C^{0}} \int_{\mathbb{R}^{d} \times \mathbb{R}^{d}} f^{\mathrm{in}} \mathrm{d}(x, v)+\left|\int_{\delta}^{+\infty} \int_{\mathbb{R}^{d} \times \mathbb{R}^{d}}\left(a^{\varepsilon_{k}}-a\right) \cdot \nabla_{v} \theta \mathbf{1}_{\left\{|v| \leq R_{0}\right\}} f^{\varepsilon_{k}} \mathrm{~d}(x, v) \mathrm{d} t\right| \\
& \quad+\left|\int_{\delta}^{+\infty} \int_{\mathbb{R}^{d} \times \mathbb{R}^{d}} a \cdot \nabla_{v} \theta\left(f^{\varepsilon_{k}}-f\right) \mathrm{d}(x, v) \mathrm{d} t\right| .
\end{aligned}
$$

We keep $\delta>0$ fixed and we pass to the limit when $k$ goes to infinity. Lemma 4.2 implies that the second term in the last right hand side can be estimated as $\left\|a^{\varepsilon_{k}}-a\right\|_{L^{\infty}\left(\mathbb{R}^{d} \times B_{R_{0}}\right)}=\left\|a_{f^{\varepsilon} k}-a_{f}\right\|_{L^{\infty}\left(\mathbb{R}^{d} \times B_{R_{0}}\right)} \leq C\left(R_{0}\right) W_{1}\left(f^{\varepsilon_{k}}(t), f(t)\right) \rightarrow 0$ when $k \rightarrow+\infty$ 
uniformly for $t \in[\delta, T]$, implying, for $T$ large enough

$$
\left|\int_{\delta}^{+\infty} \int_{|v| \leq R_{0}}\left(a^{\varepsilon_{k}}-a\right) \cdot \nabla_{v} \theta f^{\varepsilon_{k}} \mathrm{~d}(x, v) \mathrm{d} t\right| \leq C\left(R_{0}\right)\|\theta\|_{C^{1}} \int_{\delta}^{T} W_{1}\left(f^{\varepsilon_{k}}(t), f(t)\right) \mathrm{d} t \rightarrow 0
$$

when $k$ goes to infinity. For the third term in the right hand side of (24) we use the weak $\star$ convergence $\lim _{k \rightarrow+\infty} f^{\varepsilon_{k}}(t)=f(t)$ in $\mathcal{M}_{b}^{+}\left(\mathbb{R}^{d} \times \mathbb{R}^{d}\right)$ for any $t \geq \delta$, cf. Proposition 2.1

$$
\lim _{k \rightarrow+\infty} \int_{\mathbb{R}^{d} \times \mathbb{R}^{d}} a \cdot \nabla_{v} \theta\left(f^{\varepsilon_{k}}(t)-f(t)\right) \mathrm{d}(x, v)=0, \quad t \geq \delta
$$

and we conclude by the Lebesgue dominated convergence theorem

$$
\lim _{k \rightarrow+\infty} \int_{\delta}^{+\infty} \int_{\mathbb{R}^{d} \times \mathbb{R}^{d}} a \cdot \nabla_{v} \theta\left(f^{\varepsilon_{k}}(t, x, v)-f(t, x, v)\right) \mathrm{d}(x, v) \mathrm{d} t=0 .
$$

Passing to the limit in (24) when $k$ goes to infinity, we obtain

$$
\limsup _{k \rightarrow+\infty}\left|\int_{\mathbb{R}_{+}} \int_{\mathbb{R}^{d} \times \mathbb{R}^{d}}\left\{a^{\varepsilon_{k}} \cdot \nabla_{v} \theta f^{\varepsilon_{k}}-a \cdot \nabla_{v} \theta f\right\} \mathrm{d}(x, v) \mathrm{d} t\right| \leq 2 A \delta\left\|\nabla_{v} \theta\right\|_{C^{0}}
$$

Sending $\delta$ to 0 we obtain that

$$
\lim _{k \rightarrow+\infty} \int_{\mathbb{R}_{+}} \int_{\mathbb{R}^{d} \times \mathbb{R}^{d}} a^{\varepsilon_{k}} \cdot \nabla_{v} \theta f^{\varepsilon_{k}} \mathrm{~d}(x, v) \mathrm{d} t=\int_{\mathbb{R}_{+}} \int_{\mathbb{R}^{d} \times \mathbb{R}^{d}} a \cdot \nabla_{v} \theta f \mathrm{~d}(x, v) \mathrm{d} t .
$$

\section{Diffusion models}

We intend to introduce a formalism which will allow us to investigate in a simpler manner the asymptotic behavior of (11) and (9). This method comes from gyrokinetic models in plasma physics: when studying the magnetic confinement we are looking for averaged models with respect to the fast motion of particles around the magnetic lines. The analysis relies on the notion of gyro-average operator [6], which is a projection onto the space of slow time depending functions. In other words, projecting means smoothing out the fluctuations with respect to the fast time variable, corresponding to the high cyclotronic frequency. This projection appears like a gyro-average operator. Here the arguments are developed at a formal level.

We first introduce rigorously the projected measure on the sphere $r \mathbb{S}$ for general measures. Let $f \in \mathcal{M}_{b}^{+}\left(\mathbb{R}^{d} \times \mathbb{R}^{d}\right)$ be a non negative bounded measure on $\mathbb{R}^{d} \times \mathbb{R}^{d}$. We denote by $\langle f\rangle$ the measure corresponding to the linear application

$$
\psi \rightarrow \int_{\mathbb{R}^{d} \times \mathbb{R}^{d}} \psi(x, v) \mathbf{1}_{v=0} f(x, v) \mathrm{d}(x, v)+\int_{\mathbb{R}^{d} \times \mathbb{R}^{d}} \psi\left(x, r \frac{v}{|v|}\right) \mathbf{1}_{v \neq 0} f(x, v) \mathrm{d}(x, v),
$$


for all $\psi \in C_{c}^{0}\left(\mathbb{R}^{d} \times \mathbb{R}^{d}\right)$, i.e.,

$\int_{\mathbb{R}^{d} \times \mathbb{R}^{d}} \psi(x, v)\langle f\rangle(x, v) \mathrm{d}(x, v)=\int_{v=0} \psi(x, v) f(x, v) \mathrm{d}(x, v)+\int_{v \neq 0} \psi\left(x, r \frac{v}{|v|}\right) f(x, v) \mathrm{d}(x, v)$, for all $\psi \in C_{c}^{0}\left(\mathbb{R}^{d} \times \mathbb{R}^{d}\right)$. Observe that $\langle f\rangle$ is a non negative bounded measure,

$$
\int_{\mathbb{R}^{d} \times \mathbb{R}^{d}}\langle f\rangle(x, v) \mathrm{d}(x, v)=\int_{\mathbb{R}^{d} \times \mathbb{R}^{d}} f(x, v) \mathrm{d}(x, v),
$$

with supp $\langle f\rangle \subset \mathbb{R}^{d} \times(\{0\} \cup r \mathbb{S})$. We have the following characterization.

Proposition 5.1 Assume that $f$ is a non negative bounded measure on $\mathbb{R}^{d} \times \mathbb{R}^{d}$. Then $\langle f\rangle$ is the unique measure $F$ satisfying $\operatorname{supp} F \subset \mathbb{R}^{d} \times(\{0\} \cup r \mathbb{S})$,

$$
\int_{v \neq 0} \psi\left(x, r \frac{v}{|v|}\right) F(x, v) \mathrm{d}(x, v)=\int_{v \neq 0} \psi\left(x, r \frac{v}{|v|}\right) f(x, v) \mathrm{d}(x, v), \quad \psi \in C_{c}^{0}\left(\mathbb{R}^{d} \times \mathbb{R}^{d}\right)
$$

and $F=f$ on $\mathbb{R}^{d} \times\{0\}$.

Proof. The measure $\langle f\rangle$ defined before satisfies the above characterization. Indeed, supp $\langle f\rangle \subset$ $\mathbb{R}^{d} \times(\{0\} \cup r \mathbb{S})$. Taking now $\psi(x, v)=\varphi(x) \chi(|v| / \delta)$ with $\varphi \in C_{c}^{0}\left(\mathbb{R}^{d}\right)$ and $\delta>0$ one gets

$$
\begin{aligned}
\int_{\mathbb{R}^{d} \times \mathbb{R}^{d}} \varphi(x) \chi\left(\frac{|v|}{\delta}\right)\langle f\rangle(x, v) \mathrm{d}(x, v)= & \int_{v=0} \varphi(x) f(x, v) \mathrm{d}(x, v) \\
& +\int_{v \neq 0} \varphi(x) \chi\left(\frac{|v|}{\delta}\right) f(x, v) \mathrm{d}(x, v) .
\end{aligned}
$$

Passing to the limit for $\delta \searrow 0$ yields

$$
\int_{v=0} \varphi(x)\langle f\rangle(x, v) \mathrm{d}(x, v)=\int_{v=0} \varphi(x) f(x, v) \mathrm{d}(x, v), \quad \varphi \in C_{c}^{0}\left(\mathbb{R}^{d}\right)
$$

meaning that $\langle f\rangle=f$ on $\mathbb{R}^{d} \times\{0\}$. Therefore one gets for any $\psi \in C_{c}^{0}\left(\mathbb{R}^{d} \times \mathbb{R}^{d}\right)$

$$
\begin{aligned}
\int_{v \neq 0} \psi\left(x, r \frac{v}{|v|}\right)\langle f\rangle(x, v) \mathrm{d}(x, v) & =\int_{|v|=r} \psi(x, v)\langle f\rangle(x, v) \mathrm{d}(x, v) \\
& =\int_{v \neq 0} \psi(x, v)\langle f\rangle(x, v) \mathrm{d}(x, v) \\
& =\int_{\mathbb{R}^{d} \times \mathbb{R}^{d}} \psi\langle f\rangle(x, v) \mathrm{d}(x, v)-\int_{v=0} \psi\langle f\rangle(x, v) \mathrm{d}(x, v) \\
& =\int_{\mathbb{R}^{d} \times \mathbb{R}^{d}} \psi\langle f\rangle(x, v) \mathrm{d}(x, v)-\int_{v=0} \psi f(x, v) \mathrm{d}(x, v) \\
& =\int_{v \neq 0} \psi\left(x, r \frac{v}{|v|}\right) f(x, v) \mathrm{d}(x, v) .
\end{aligned}
$$


Conversely, let us check that the above characterization exactly defines the measure $\langle f\rangle$. For any $\psi \in C_{c}^{0}\left(\mathbb{R}^{d} \times \mathbb{R}^{d}\right)$ we have

$$
\begin{aligned}
\int_{\mathbb{R}^{d} \times \mathbb{R}^{d}} \psi(x, v) F(x, v) \mathrm{d}(x, v) & =\int_{v=0} \psi F(x, v) \mathrm{d}(x, v)+\int_{v \neq 0} \psi F(x, v) \mathrm{d}(x, v) \\
& =\int_{v=0} \psi(x, v) f(x, v) \mathrm{d}(x, v)+\int_{v \neq 0} \psi\left(x, r \frac{v}{|v|}\right) F(x, v) \mathrm{d}(x, v) \\
& =\int_{v=0} \psi(x, v) f(x, v) \mathrm{d}(x, v)+\int_{v \neq 0} \psi\left(x, r \frac{v}{|v|}\right) f(x, v) \mathrm{d}(x, v)
\end{aligned}
$$

saying that $F=\langle f\rangle$.

By Proposition 5.1 it is clear that $\langle\cdot\rangle$ leaves invariant the measures with support in $\mathbb{R}^{d} \cup(\{0\} \cup r \mathbb{S})$. Consider $f \in \mathcal{M}_{b}^{+}\left(\mathbb{R}^{d} \times \mathbb{R}^{d}\right)$. We say that $\operatorname{div}_{v}\left\{f\left(\alpha-\beta|v|^{2}\right) v\right\} \in \mathcal{M}_{b}\left(\mathbb{R}^{d} \times \mathbb{R}^{d}\right)$ if and only if there is a constant $C>0$ such that

$$
\int_{\mathbb{R}^{d} \times \mathbb{R}^{d}}\left(\alpha-\beta|v|^{2}\right) v \cdot \nabla_{v} \psi f(x, v) \mathrm{d}(x, v) \leq C\|\psi\|_{L^{\infty}}, \quad \psi \in C_{c}^{1}\left(\mathbb{R}^{d} \times \mathbb{R}^{d}\right) .
$$

In this case there is a bounded measure $\mu$ such that

$$
-\int_{\mathbb{R}^{d} \times \mathbb{R}^{d}}\left(\alpha-\beta|v|^{2}\right) v \cdot \nabla_{v} \psi f(x, v) \mathrm{d}(x, v)=\int_{\mathbb{R}^{d} \times \mathbb{R}^{d}} \psi \mu, \quad \psi \in C_{c}^{1}\left(\mathbb{R}^{d} \times \mathbb{R}^{d}\right) .
$$

By definition we take $\operatorname{div}_{v}\left\{f\left(\alpha-\beta|v|^{2}\right) v\right\}=\mu$. The main motivation for the construction of the projection $\langle\cdot\rangle$ is the following result.

Proposition 5.2 For any $f \in \mathcal{M}_{b}^{+}\left(\mathbb{R}^{d} \times \mathbb{R}^{d}\right)$ such that $\operatorname{div}_{v}\left\{f\left(\alpha-\beta|v|^{2}\right) v\right\} \in \mathcal{M}_{b}\left(\mathbb{R}^{d} \times \mathbb{R}^{d}\right)$ we have $\left\langle\operatorname{div}_{v}\left\{f\left(\alpha-\beta|v|^{2}\right) v\right\}\right\rangle=0$.

Proof. Let us take $\operatorname{div}_{v}\left\{f\left(\alpha-\beta|v|^{2}\right) v\right\}=\mu$. We will check that the zero measure 0 satisfies the characterization of $\langle\mu\rangle$ in Proposition 5.1. Clearly supp $0=\emptyset \subset \mathbb{R}^{d} \times(\{0\} \cup r \mathbb{S})$. For any $\varphi(x) \in C_{c}^{0}\left(\mathbb{R}^{d}\right)$ we have

$$
\begin{aligned}
\int_{v=0} \varphi(x) \mu(x, v) \mathrm{d}(x, v) & =\lim _{\delta \searrow 0} \int_{\mathbb{R}^{d} \times \mathbb{R}^{d}} \varphi(x) \chi\left(\frac{|v|}{\delta}\right) \mu(x, v) \mathrm{d}(x, v) \\
& =-\lim _{\delta \searrow 0} \int_{\mathbb{R}^{d} \times \mathbb{R}^{d}} \varphi(x) \chi^{\prime}\left(\frac{|v|}{\delta}\right) \frac{|v|}{\delta}\left(\alpha-\beta|v|^{2}\right) f(x, v) \mathrm{d}(x, v)=0
\end{aligned}
$$

by dominated convergence, since

$$
\left|\chi^{\prime}\left(\frac{|v|}{\delta}\right) \frac{|v|}{\delta}\left(\alpha-\beta|v|^{2}\right)\right| \leq \alpha \sup _{u \geq 0}\left|\chi^{\prime}(u) u\right|+\beta \delta^{2} \sup _{u \geq 0}\left|\chi^{\prime}(u) u^{3}\right| .
$$

Therefore we deduce that $\operatorname{div}_{v}\left\{f\left(\alpha-\beta|v|^{2}\right) v\right\}=0$ on $\mathbb{R}^{d} \times\{0\}$. Consider now $\psi \in C_{c}^{1}\left(\mathbb{R}^{d} \times \mathbb{R}^{d}\right)$ and lets us compute

$$
\begin{array}{r}
\int_{v \neq 0} \psi\left(x, r \frac{v}{|v|}\right) \mu(x, v) \mathrm{d}(x, v)=\lim _{\delta \searrow 0} \int_{\mathbb{R}^{d} \times \mathbb{R}^{d}} \psi\left(x, r \frac{v}{|v|}\right)\left(1-\chi\left(\frac{|v|}{\delta}\right)\right) \mu(x, v) \mathrm{d}(x, v) \\
=\lim _{\delta \searrow 0} \int_{\mathbb{R}^{d} \times \mathbb{R}^{d}} \psi\left(x, r \frac{v}{|v|}\right) \chi^{\prime}\left(\frac{|v|}{\delta}\right) \frac{|v|}{\delta}\left(\alpha-\beta|v|^{2}\right) f(x, v) \mathrm{d}(x, v)=0
\end{array}
$$


since $v \cdot \nabla_{v}\left\{\psi\left(x, r \frac{v}{|v|}\right)\right\}=0$. By density, the same conclusion holds true for any $\psi \in C_{c}^{0}\left(\mathbb{R}^{d} \times\right.$ $\left.\mathbb{R}^{d}\right)$ and thus $\left\langle\operatorname{div}_{v}\left\{f\left(\alpha-\beta|v|^{2}\right) v\right\}\right\rangle=0$.

Remark 5.1 When $f \in \mathcal{M}_{b}^{+}\left(\mathbb{R}^{d} \times \mathbb{R}^{d}\right)$ does not charge $\mathbb{R}^{d} \times\{0\}$, $\langle f\rangle$ is given by

$$
\operatorname{supp}\langle f\rangle \subset \mathbb{R}^{d} \times r \mathbb{S}, \quad \int_{v \neq 0} \psi\left(x, r \frac{v}{|v|}\right)\langle f\rangle=\int_{v \neq 0} \psi\left(x, r \frac{v}{|v|}\right) f, \quad \psi \in C_{c}^{0}\left(\mathbb{R}^{d} \times \mathbb{R}^{d}\right)
$$

or equivalently

$$
\int_{\mathbb{R}^{d} \times \mathbb{R}^{d}} \psi\langle f\rangle=\int_{v \neq 0} \psi\left(x, r \frac{v}{|v|}\right) f, \quad \psi \in C_{c}^{0}\left(\mathbb{R}^{d} \times \mathbb{R}^{d}\right)
$$

Using Proposition 5.2 we can obtain, at least formally, the limit model satisfied by $f=$ $\lim _{\varepsilon \searrow 0} f^{\varepsilon}$. By (44) we know that $\operatorname{supp} f \subset \mathbb{R}^{d} \times(\{0\} \cup r \mathbb{S})$. The time evolution of $f$ comes by eliminating $f^{(1)}$ in (5). For that it is sufficient to project on the subspace of the measures satisfying the constraint (4), i.e., to apply $\langle\cdot\rangle$.

$$
\left\langle\partial_{t} f\right\rangle+\left\langle\operatorname{div}_{x}\{f v\}\right\rangle+\left\langle\operatorname{div}_{v}\{f a\}\right\rangle=0 .
$$

It is easily seen that $\left\langle\partial_{t} f\right\rangle=\partial_{t}\langle f\rangle=\partial_{t} f$ since $\operatorname{supp} f \subset \mathbb{R}^{d} \times(\{0\} \cup r \mathbb{S})$ and therefore $\langle f\rangle=f$. We need to compute the last two terms in (26). We show that

Proposition 5.3 Assume that $a=a(x)$ is a bounded continuous field. Then we have the following equalities

$$
\begin{gathered}
\left\langle\operatorname{div}_{x}\{f v\}\right\rangle=\operatorname{div}_{x}\{f v\} \quad \text { if } \operatorname{supp} f \subset \mathbb{R}^{d} \times(\{0\} \cup r \mathbb{S}) \\
\left\langle\operatorname{div}_{v}\{f a\}\right\rangle=\operatorname{div}_{v}\left\{f\left(I-\frac{v \otimes v}{|v|^{2}}\right) a\right\} \quad \text { if } \operatorname{supp} f \subset \mathbb{R}^{d} \times r \mathbb{S} .
\end{gathered}
$$

As a consequence, (26) yields the transport equation (6) obtained rigorously in Theorems 1.1 and 1.2 .

Proof. For any $\psi \in C_{c}^{1}\left(\mathbb{R}^{d} \times \mathbb{R}^{d}\right)$ we have

$$
\begin{aligned}
\int_{\mathbb{R}^{d} \times \mathbb{R}^{d}} \psi & \left\langle\operatorname{div}_{x}\{f v\}\right\rangle=\int_{v=0} \psi \operatorname{div}_{x}\{f v\}+\int_{v \neq 0} \psi\left(x, r \frac{v}{|v|}\right) \operatorname{div}_{x}\{f v\} \\
& =\lim _{\delta \searrow 0} \int_{\mathbb{R}^{d} \times \mathbb{R}^{d}} \psi \chi\left(\frac{|v|}{\delta}\right) \operatorname{div}_{x}\{f v\}+\lim _{\delta \searrow 0} \int_{\mathbb{R}^{d} \times \mathbb{R}^{d}} \psi\left(x, r \frac{v}{|v|}\right)\left(1-\chi\left(\frac{|v|}{\delta}\right)\right) \operatorname{div}_{x}\{f v\} \\
& =-\lim _{\delta \searrow 0} \int_{\mathbb{R}^{d} \times \mathbb{R}^{d}} v \cdot \nabla_{x} \psi \chi\left(\frac{|v|}{\delta}\right) f-\lim _{\delta \searrow 0} \int_{\mathbb{R}^{d} \times \mathbb{R}^{d}} v \cdot \nabla_{x} \psi\left(x, r \frac{v}{|v|}\right)\left(1-\chi\left(\frac{|v|}{\delta}\right)\right) f \\
& =-\int_{v=0} v \cdot \nabla_{x} \psi f-\int_{v \neq 0} v \cdot \nabla_{x} \psi\left(x, r \frac{v}{|v|}\right) f \\
& =-\int_{\mathbb{R}^{d} \times \mathbb{R}^{d}} v \cdot \nabla_{x} \psi f=\int_{\mathbb{R}^{d} \times \mathbb{R}^{d}} \psi \operatorname{div}_{x}\{f v\}
\end{aligned}
$$


saying that $\left\langle\operatorname{div}_{x}\{f v\}\right\rangle=\operatorname{div}_{x}\{f v\}$. Assume now that supp $f \subset \mathbb{R}^{d} \times r \mathbb{S}$. It is easily seen that $\operatorname{div}_{v}(f a)$ does not charge $\mathbb{R}^{d} \times\{0\}$. Indeed, for any $\psi \in C_{c}^{0}\left(\mathbb{R}^{d} \times \mathbb{R}^{d}\right)$ we have by dominated convergence

$$
\begin{aligned}
\int_{v=0} \psi \operatorname{div}_{v}(f a) & =\lim _{\delta \searrow 0} \int_{\mathbb{R}^{d} \times \mathbb{R}^{d}} \psi \chi\left(\frac{|v|}{\delta}\right) \operatorname{div}_{v}(f a) \\
& =-\lim _{\delta \searrow 0} \int_{\mathbb{R}^{d} \times \mathbb{R}^{d}} a \cdot \nabla_{v} \psi \chi\left(\frac{|v|}{\delta}\right) f-\lim _{\delta \searrow 0} \int_{\mathbb{R}^{d} \times \mathbb{R}^{d}} a \cdot \frac{v}{|v|} \frac{1}{\delta} \chi^{\prime}\left(\frac{|v|}{\delta}\right) \psi f=0 .
\end{aligned}
$$

Therefore we can use (25)

$$
\begin{aligned}
\int_{\mathbb{R}^{d} \times \mathbb{R}^{d}} \psi\left\langle\operatorname{div}_{v}(f a)\right\rangle= & \int_{v \neq 0} \psi\left(x, r \frac{v}{|v|}\right) \operatorname{div}_{v}(f a) \\
= & \lim _{\delta \searrow 0} \int_{\mathbb{R}^{d} \times \mathbb{R}^{d}}\left(1-\chi\left(\frac{|v|}{\delta}\right)\right) \psi\left(x, r \frac{v}{|v|}\right) \operatorname{div}_{v}(f a) \\
= & -\lim _{\delta \searrow 0} \int_{\mathbb{R}^{d} \times \mathbb{R}^{d}}\left(1-\chi\left(\frac{|v|}{\delta}\right)\right) \frac{r}{|v|}\left(I-\frac{v \otimes v}{|v|^{2}}\right) a \cdot\left(\nabla_{v} \psi\right)\left(x, r \frac{v}{|v|}\right) f \\
& +\lim _{\delta \searrow 0} \int_{\mathbb{R}^{d} \times \mathbb{R}^{d}} \frac{1}{\delta} \chi^{\prime}\left(\frac{|v|}{\delta}\right) \frac{v}{|v|} \cdot a \psi\left(x, r \frac{v}{|v|}\right) f \\
= & -\int_{v \neq 0}\left(I-\frac{v \otimes v}{|v|^{2}}\right) a \cdot \nabla_{v} \psi f=\int_{\mathbb{R}^{d} \times \mathbb{R}^{d}} \psi \operatorname{div}_{v}\left\{f\left(I-\frac{v \otimes v}{|v|^{2}}\right) a\right\} .
\end{aligned}
$$

We investigate now the limit when $\varepsilon \searrow 0$ of the diffusion model (9). We are done if we compute $\left\langle\Delta_{v} f\right\rangle$ for a non negative bounded measure with support contained in $\mathbb{R}^{d} \times r \mathbb{S}$. As before we can check that $\Delta_{v} f$ does not charge $\mathbb{R}^{d} \times\{0\}$ and therefore, thanks to (25), we obtain after some computations

$$
\int_{\mathbb{R}^{d} \times \mathbb{R}^{d}} \psi\left\langle\Delta_{v} f\right\rangle=\int_{v \neq 0} \psi\left(x, r \frac{v}{|v|}\right) \Delta_{v} f=\int_{v \neq 0} \Delta_{v}\left\{\psi\left(x, r \frac{v}{|v|}\right)\right\} f, \quad \psi \in C_{c}^{2}\left(\mathbb{R}^{d} \times \mathbb{R}^{d}\right) .
$$

Lemma 5.1 For any function $\varphi \in C^{2}\left(\mathbb{R}^{d} \backslash\{0\}\right)$ and any $r>0$ we have

$$
\Delta_{v}\left\{\varphi\left(r \frac{v}{|v|}\right)\right\}=\left(\frac{r}{|v|}\right)^{2}\left(I-\frac{v \otimes v}{|v|^{2}}\right): \partial_{v}^{2} \varphi\left(r \frac{v}{|v|}\right)-2 \frac{r}{|v|} \frac{v \cdot \nabla_{v} \varphi\left(r \frac{v}{|v|}\right)}{|v|^{2}}, v \neq 0 .
$$

Combining (27), Lemma 5.1 and the fact that supp $f \subset \mathbb{R}^{d} \times r \mathbb{S}$ we obtain

$$
\begin{aligned}
\int_{\mathbb{R}^{d} \times \mathbb{R}^{d}} \psi(x, v)\left\langle\Delta_{v} f\right\rangle & =\int_{v \neq 0}\left[\left(I-\frac{v \otimes v}{|v|^{2}}\right): \partial_{v}^{2} \psi(x, v)-2 \frac{v \cdot \nabla_{v} \psi(x, v)}{|v|^{2}}\right] f \\
& =\int_{\mathbb{R}^{d} \times \mathbb{R}^{d}} \psi(x, v) \operatorname{div}_{v}\left\{\operatorname{div}_{v}\left[f\left(I-\frac{v \otimes v}{|v|^{2}}\right)\right]+2 f \frac{v}{|v|^{2}}\right\} .
\end{aligned}
$$


We deduce the formula

$$
\left\langle\Delta_{v} f\right\rangle=\operatorname{div}_{v}\left\{\operatorname{div}_{v}\left[f\left(I-\frac{v \otimes v}{|v|^{2}}\right)\right]+2 f \frac{v}{|v|^{2}}\right\}
$$

for any $f$ satisfying supp $f \subset \mathbb{R}^{d} \times r \mathbb{S}$ and the limit of the Vicsek model (9) when $\varepsilon \searrow 0$ becomes

$$
\partial_{t} f+\operatorname{div}_{x}(f v)+\operatorname{div}_{v}\left\{f\left(I-\frac{v \otimes v}{|v|^{2}}\right) a\right\}=\operatorname{div}_{v}\left\{\operatorname{div}_{v}\left[f\left(I-\frac{v \otimes v}{|v|^{2}}\right)\right]+2 f \frac{v}{|v|^{2}}\right\}
$$

with the initial condition $f(0)=\left\langle f^{\text {in }}\right\rangle$, as stated in (10).

\section{A Spherical coordinates and the Laplace-Beltrami operator}

In this appendix, we show the computations to relate the equations written in original variables $(x, v)$ to the equations in spherical coordinates $(x, \omega)$. Our limit densities have their support contained in $\mathbb{R}^{d} \times r \mathbb{S}$ and thus reduce to measures on $\mathbb{R}^{d} \times r \mathbb{S}$. For example, let us consider the measure on $\mathbb{R}^{d} \times r \mathbb{S}$ still denoted by $f$, given by

$$
\int_{\mathbb{R}^{d} \times r \mathbb{S}} \psi(x, \omega) f(x, \omega) \mathrm{d}(x, \omega)=\int_{v \neq 0} \psi\left(x, r \frac{v}{|v|}\right) f(x, v) \mathrm{d}(x, v)
$$

for any function $\psi \in C_{c}^{0}\left(\mathbb{R}^{d} \times r \mathbb{S}\right)$. In particular, to any $f \in \mathcal{M}_{b}^{+}\left(\mathbb{R}^{d} \times \mathbb{R}^{d}\right)$ not charging $\mathbb{R}^{d} \times\{0\}$ it corresponds $\langle f\rangle \in \mathcal{M}_{b}^{+}\left(\mathbb{R}^{d} \times \mathbb{R}^{d}\right)$, with supp $\langle f\rangle \subset \mathbb{R}^{d} \times r \mathbb{S}$, whose characterization is

$$
\int_{\mathbb{R}^{d} \times r \mathbb{S}} \psi(x, \omega)\langle f\rangle(x, \omega) \mathrm{d}(x, \omega)=\int_{v \neq 0} \psi\left(x, r \frac{v}{|v|}\right) f(x, v) \mathrm{d}(x, v) .
$$

We intend to write the previous limit models (in Theorems 1.1, 1.2, and (28) ) in spherical coordinates.

Proposition A.1 Assume that $f \in \mathcal{M}_{b}^{+}\left(\mathbb{R}^{d} \times \mathbb{R}^{d}\right)$, supp $f \subset \mathbb{R}^{d} \times r \mathbb{S}$ and let us denote by $F \in \mathcal{M}_{b}^{+}\left(\mathbb{R}^{d} \times r \mathbb{S}\right)$ its corresponding measure on $\mathbb{R}^{d} \times r \mathbb{S}$. Therefore we have

$$
\left\langle\operatorname{div}_{x}(f v)\right\rangle=\operatorname{div}_{x}(F \omega),\left\langle\operatorname{div}_{v}(f a)\right\rangle=\operatorname{div}_{\omega}\left\{F\left(I-\frac{1}{r^{2}}(\omega \otimes \omega)\right) a\right\},\left\langle\Delta_{v} f\right\rangle=\Delta_{\omega} F
$$

Proof. Thanks to Proposition 5.3 we have for any $\psi \in C_{c}^{1}\left(\mathbb{R}^{d} \times r \mathbb{S}\right)$

$$
\begin{aligned}
\int_{\mathbb{R}^{d} \times r \mathbb{S}} \psi(x, \omega)\left\langle\operatorname{div}_{x}(f v)\right\rangle & =\int_{v \neq 0} \psi\left(x, r \frac{v}{|v|}\right) \operatorname{div}_{x}(f v)=-\int_{v \neq 0} v \cdot \nabla_{x} \psi\left(x, r \frac{v}{|v|}\right) f \\
& =-\int_{v \neq 0} r \frac{v}{|v|} \cdot \nabla_{x} \psi\left(x, r \frac{v}{|v|}\right) f=-\int_{\mathbb{R}^{d} \times r \mathbb{S}} \omega \cdot \nabla_{x} \psi(x, \omega) F
\end{aligned}
$$


and thus $\left\langle\operatorname{div}_{x}(f v)\right\rangle=\operatorname{div}_{x}(F \omega)$. Similarly we can write

$$
\begin{aligned}
\int_{\mathbb{R}^{d} \times r \mathbb{S}} \psi(x, \omega)\left\langle\operatorname{div}_{v}(f a)\right\rangle & =\int_{v \neq 0} \psi\left(x, r \frac{v}{|v|}\right)\left\langle\operatorname{div}_{v}(f a)\right\rangle(\mathrm{d}(x, v)) \\
& =\int_{v \neq 0} \psi\left(x, r \frac{v}{|v|}\right) \operatorname{div}_{v}\left\{f\left(I-\frac{v \otimes v}{|v|^{2}}\right) a\right\} \\
& =-\int_{v \neq 0} \frac{r}{|v|}\left(I-\frac{v \otimes v}{|v|^{2}}\right) a \cdot\left(I-\frac{v \otimes v}{|v|^{2}}\right) \nabla_{v} \psi\left(x, r \frac{v}{|v|}\right) f \\
& =-\int_{v \neq 0}\left(I-\frac{v \otimes v}{|v|^{2}}\right) a \cdot\left(I-\frac{v \otimes v}{|v|^{2}}\right) \nabla_{v} \psi\left(x, r \frac{v}{|v|}\right) f \\
& =-\int_{\mathbb{R}^{d} \times r \mathbb{S}}\left(I-\frac{1}{r^{2}}(\omega \otimes \omega)\right) a \cdot\left(I-\frac{1}{r^{2}}(\omega \otimes \omega)\right) \nabla_{v} \psi(x, \omega) F \\
& =-\int_{\mathbb{R}^{d} \times r \mathbb{S}}\left(I-\frac{1}{r^{2}}(\omega \otimes \omega)\right) a \cdot \nabla_{\omega} \psi(x, \omega) F \\
& =\int_{\mathbb{R}^{d} \times r \mathbb{S}} \psi(x, \omega) \operatorname{div}_{\omega}\left\{F\left(I-\frac{1}{r^{2}}(\omega \otimes \omega)\right) a\right\}
\end{aligned}
$$

and therefore

$$
\left\langle\operatorname{div}_{v}(f a)\right\rangle=\operatorname{div}_{\omega}\left\{F\left(I-\frac{1}{r^{2}}(\omega \otimes \omega)\right) a\right\} .
$$

Here $\operatorname{div}_{\omega}$ stands for the divergence along $r \mathbb{S}$ (notice that $\left(I-\frac{1}{r^{2}}(\omega \otimes \omega)\right) a$ is a tangent field of $r \mathbb{S})$ and $\nabla_{\omega}=\left(I-\frac{1}{r^{2}}(\omega \otimes \omega)\right) \nabla_{v}$ is the gradient along $r \mathbb{S}$. For the last assertion we appeal to the following well known result asserting that the Laplace-Beltrami operator coincides with the Laplacian of the degree zero homogeneous extension, see also [4].

Proposition A.2 Consider $\varphi=\varphi(\omega)$ a $C^{2}$ function on $r \mathbb{S}$ and we denote by $\Phi=\Phi(v)$ its degree zero homogeneous extension on $\mathbb{R}^{d} \backslash\{0\}$

$$
\Phi(v)=\varphi\left(r \frac{v}{|v|}\right), \quad v \neq 0 .
$$

Therefore we have for any $\omega \in r \mathbb{S}$

$$
\Delta_{\omega} \varphi(\omega)=\Delta_{v} \Phi(\omega)
$$

Let us come back to the proof of Proposition A.1. For any $\psi \in C_{c}^{2}\left(\mathbb{R}^{d} \times r \mathbb{S}\right)$ we introduce its degree zero homogeneous extension $\Psi(x, v)=\psi\left(x, r \frac{v}{|v|}\right)$. Thanks to Proposition A.2 we can write

$$
\begin{aligned}
\int_{\mathbb{R}^{d} \times r \mathbb{S}} \psi(x, \omega)\left\langle\Delta_{v} f\right\rangle & =\int_{v \neq 0} \psi\left(x, r \frac{v}{|v|}\right)\left\langle\Delta_{v} f\right\rangle=\int_{v \neq 0} \Psi(x, v) \Delta_{v} f=\int_{v \neq 0} \Delta_{v} \Psi f \\
& =\int_{|v|=r} \Delta_{\omega} \psi(x, v) f=\int_{\mathbb{R}^{d} \times r \mathbb{S}} \Delta_{\omega} \psi(x, \omega) F=\int_{\mathbb{R}^{d} \times r \mathbb{S}} \psi(x, \omega) \Delta_{\omega} F
\end{aligned}
$$

meaning that $\left\langle\Delta_{v} f\right\rangle=\Delta_{\omega} F$. 
For the sake of completeness, we finally write the equations in spherical coordinates in $\mathbb{R}^{3}$. We introduce the spherical coordinates $\omega=r(\cos \theta \cos \varphi, \cos \theta \sin \varphi, \sin \theta)$ with the angle variables $(\theta, \varphi) \in]-\pi / 2, \pi / 2[\times[0,2 \pi[$, and the orthogonal basis of the tangent space to $r \mathbb{S}$

$$
e_{\theta}=(-\sin \theta \cos \varphi,-\sin \theta \sin \varphi, \cos \theta), \quad e_{\varphi}=(-\cos \theta \sin \varphi, \cos \theta \cos \varphi, 0)
$$

with $\left|e_{\theta}\right|=1,\left|e_{\varphi}\right|=\cos \theta$. For any smooth function $u$ on $r \mathbb{S}$ we have

$$
\nabla_{\omega} u=\left(\nabla_{\omega} u \cdot e_{\theta}\right) e_{\theta}+\left(\nabla_{\omega} u \cdot e_{\varphi}\right) \frac{e_{\varphi}}{\cos ^{2} \theta}=\frac{1}{r} \partial_{\theta} u e_{\theta}+\frac{1}{r \cos ^{2} \theta} \partial_{\varphi} u e_{\varphi}
$$

and for any smooth tangent field $\xi=\xi_{\theta} e_{\theta}+\xi_{\varphi} e_{\varphi}$ we have

$$
\operatorname{div}_{\omega} \xi=\frac{1}{r}\left\{\frac{1}{\cos \theta} \partial_{\theta}\left(\xi_{\theta} \cos \theta\right)+\partial_{\varphi} \xi_{\varphi}\right\}
$$

The coordinates of the tangent field $\xi:=F\left(I-\frac{1}{r^{2}}(\omega \otimes \omega)\right) a$ are $\xi_{\theta}=\xi \cdot e_{\theta}=F a_{\theta}, \quad \xi_{\varphi}=$ $\frac{\xi \cdot e_{\varphi}}{\cos ^{2} \theta}=F a_{\varphi}$ and we obtain

$$
\left\langle\operatorname{div}_{v}(f a)\right\rangle=\operatorname{div}_{\omega}\left\{F\left(I-\frac{1}{r^{2}}(\omega \otimes \omega)\right) a\right\}=\frac{1}{r}\left\{\frac{1}{\cos \theta} \partial_{\theta}\left(F a_{\theta} \cos \theta\right)+\partial_{\varphi}\left(F a_{\varphi}\right)\right\} .
$$

The spherical Laplacian is given by

$$
\begin{aligned}
\Delta_{\omega} F & =\operatorname{div}_{\omega}\left(\nabla_{\omega} F\right)=\frac{1}{r}\left\{\frac{1}{\cos \theta} \frac{\partial}{\partial \theta}\left(\frac{\cos \theta}{r} \partial_{\theta} F\right)+\frac{\partial}{\partial \varphi}\left(\frac{1}{r \cos ^{2} \theta} \partial_{\varphi} F\right)\right\} \\
& =\frac{1}{r^{2}}\left\{\frac{1}{\cos \theta} \frac{\partial}{\partial \theta}\left(\cos \theta \partial_{\theta} F\right)+\frac{1}{\cos ^{2} \theta} \partial_{\varphi}^{2} F\right\} .
\end{aligned}
$$

Proposition A.3 The limit transport equation obtained in (28) for $\mathbb{R}^{3}$ is

$$
\partial_{t} F+\omega \cdot \nabla_{x} F+\frac{1}{r}\left\{\frac{\partial_{\theta}\left(F a_{\theta} \cos \theta\right)}{\cos \theta}+\partial_{\varphi}\left(F a_{\varphi}\right)\right\}=\frac{1}{r^{2}}\left\{\frac{1}{\cos \theta} \frac{\partial}{\partial \theta}\left(\cos \theta \partial_{\theta} F\right)+\frac{1}{\cos ^{2} \theta} \partial_{\varphi}^{2} F\right\} .
$$

We recall here the proof of Proposition A.2, It is a consequence of a more general result.

Proposition A.4 Let us consider a function $\varphi=\varphi(v) \in C^{2}\left(\mathbb{R}^{d}\right), d \geq 2$ which writes in polar coordinates $\varphi(v)=\tilde{\varphi}(\rho, \sigma), \quad \rho=|v|>0, \quad \sigma=\frac{v}{|v|} \in \mathbb{S}$. Therefore for any $v \neq 0$ we have

$$
\Delta_{v} \varphi(v)=\frac{1}{\rho^{N-1}} \frac{\partial}{\partial \rho}\left(\rho^{N-1} \partial_{\rho} \tilde{\varphi}\right)+\frac{1}{\rho^{2}} \Delta_{\sigma} \tilde{\varphi}(\rho, \sigma), \quad \rho=|v|>0, \quad \sigma=\frac{v}{|v|} .
$$

Proof. Consider a smooth function $\psi=\psi(v) \in C^{2}$ with compact support in $\mathbb{R}^{N} \backslash\{0\}$, which writes in polar coordinates $\psi(v)=\tilde{\psi}(\rho, \sigma), \quad \rho=|v|>0, \quad \sigma=\frac{v}{|v|} \in \mathbb{S}$. We have

$$
\frac{\partial \tilde{\varphi}}{\partial \rho}=\nabla_{v} \varphi \cdot \sigma, \quad \nabla_{v} \varphi=\left(\nabla_{v} \varphi \cdot \sigma\right) \sigma+(I-\sigma \otimes \sigma) \nabla_{v} \varphi=\frac{\partial \tilde{\varphi}}{\partial \rho} \sigma+\nabla_{\omega=\rho \sigma} \tilde{\varphi}
$$


and

$$
\frac{\partial \tilde{\psi}}{\partial \rho}=\nabla_{v} \psi \cdot \sigma, \quad \nabla_{v} \psi=\left(\nabla_{v} \psi \cdot \sigma\right) \sigma+(I-\sigma \otimes \sigma) \nabla_{v} \psi=\frac{\partial \tilde{\psi}}{\partial \rho} \sigma+\nabla_{\omega=\rho \sigma} \tilde{\psi}
$$

Integrating by parts yields

$$
\begin{aligned}
-\int_{\mathbb{R}^{d}} \Delta_{v} \varphi \psi(v) \mathrm{d} v & =\int_{\mathbb{R}^{d}} \nabla_{v} \varphi \cdot \nabla_{v} \psi \mathrm{d} v=\int_{\mathbb{R}_{+}} \int_{S^{N-1}}\left\{\frac{\partial \tilde{\varphi}}{\partial \rho} \frac{\partial \tilde{\psi}}{\partial \rho}+\frac{1}{\rho^{2}} \nabla_{\sigma} \tilde{\varphi} \cdot \nabla_{\sigma} \tilde{\psi}\right\} \mathrm{d} \sigma \rho^{N-1} \mathrm{~d} \rho \\
& =-\int_{S^{N-1}} \int_{\mathbb{R}_{+}} \tilde{\psi} \frac{\partial}{\partial \rho}\left(\rho^{N-1} \frac{\partial \tilde{\varphi}}{\partial \rho}\right) \mathrm{d} \rho \mathrm{d} \sigma-\int_{\mathbb{R}_{+}} \frac{\rho^{N-1}}{\rho^{2}} \int_{S^{N-1}} \tilde{\psi} \Delta_{\sigma} \tilde{\varphi} \mathrm{d} \sigma \mathrm{d} \rho \\
& =-\int_{\mathbb{R}^{d}} \psi(v)\left\{\frac{1}{\rho^{N-1}} \frac{\partial}{\partial \rho}\left(\rho^{N-1} \partial_{\rho} \tilde{\varphi}\right)+\frac{1}{\rho^{2}} \Delta_{\sigma} \tilde{\varphi}\right\} \mathrm{d} v
\end{aligned}
$$

and therefore

$$
\Delta_{v} \varphi(v)=\frac{1}{\rho^{N-1}} \frac{\partial}{\partial \rho}\left(\rho^{N-1} \partial_{\rho} \tilde{\varphi}\right)+\frac{1}{\rho^{2}} \Delta_{\sigma} \tilde{\varphi}(\rho, \sigma), \quad \rho=|v|>0, \quad \sigma=\frac{v}{|v|} .
$$

Proof. (of Proposition A.2) The degree zero homogeneous extension $\Phi(v)=\varphi\left(r \frac{v}{|v|}\right)$ does not depend on the polar radius $\Phi(v)=\tilde{\Phi}(\sigma)=\varphi(\omega=r \sigma), \quad \sigma=\frac{v}{|v|}$. Thanks to Proposition A.4, we deduce $\Delta_{v} \Phi=\frac{1}{\rho^{2}} \Delta_{\sigma} \tilde{\Phi}=\frac{r^{2}}{\rho^{2}} \Delta_{\omega} \varphi$. Taking $\rho=r$, which means $v=r \sigma=\omega$ we obtain $\Delta_{v} \Phi(\omega)=\Delta_{\omega} \varphi(\omega), \omega \in r \mathbb{S}$.

\section{Acknowledgments}

JAC was supported by projects MTM2011-27739-C04-02 and 2009-SGR-345 from Agència de Gestió d'Ajuts Universitaris i de Recerca-Generalitat de Catalunya.

\section{References}

[1] A. Barbaro, K. Taylor, P.F. Trethewey, L. Youseff, B. Birnir, Discrete and continuous models of the dynamics of pelagic fish: application to the capelin, Mathematics and Computers in Simulation 79(2009) 3397-3414.

[2] A. Barbaro, B. Einarsson, B. Birnir, S. Sigurthsson, H. Valdimarsson, O.K. Palsson, S. Sveinbjornsson, T. Sigurthsson, Modelling and simulations of the migration of pelagic fish, ICES J. Mar. Sci. 66(2009) 826-838.

[3] F. Bolley, J.A. Cañizo, J.A. Carrillo, Stochastic Mean-Field Limit: Non-Lipschitz Forces \& Swarming, Math. Mod. Meth. Appl. Sci. 21(2011) 2179-2210. 
[4] F. Bolley, J.A. Cañizo, J.A. Carrillo, Mean-field limit for the stochastic Vicsek model, Appl. Math. Letters 25(2012) 339-343.

[5] M. Bostan, The Vlasov-Poisson system with strong external magnetic field. Finite Larmor radius regime, Asymptot. Anal. 61(2009) 91-123.

[6] M. Bostan, Transport equations with disparate advection fields. Application to the gyrokinetic models in plasma physics, J. Differential Equations 249(2010) 1620-1663.

[7] M. Bostan, Gyro-kinetic Vlasov equation in three dimensional setting. Second order approximation, SIAM J. Multiscale Model. Simul. 8(2010) 1923-1957.

[8] M. Bostan, C. Negulescu, Mathematical models for strongly magnetized plasmas with mass disparate particles, Discrete Contin. Dyn. Syst. Ser. B 15(2011) 513-544.

[9] W. Braun, K. Hepp, The Vlasov dynamics and its fluctuations in the $1 / \mathrm{N}$ limit of interacting classical particles, Commun. Math. Phys. 56(1977) 101-113.

[10] J.A. Cañizo, J.A. Carrillo, J. Rosado, A well-posedness theory in measures for some kinetic models of collective motion, Math. Mod. Meth. Appl. Sci. 21(2011) 515-539.

[11] J.A. Carrillo, M.R. D’Orsogna, V. Panferov, Double milling in a self-propelled swarms from kinetic theory, Kinet. Relat. Models 2(2009) 363-378.

[12] J.A. Carrillo, M. Fornasier, J. Rosado, G. Toscani, Asymptotic flocking dynamics for the kinetic Cucker-Smale model, SIAM J. Math. Anal. 42(2010) 218-236.

[13] Carrillo, J.A., Fornasier, M., Toscani, G., Vecil, F., Particle, Kinetic, and Hydrodynamic Models of Swarming. Mathematical Modeling of Collective Behavior in SocioEconomic and Life Sciences, Series: Modelling and Simulation in Science and Technology, Birkhauser (2010), 297-336.

[14] J.A. Carrillo, A. Klar, S. Martin, S. Tiwari, Self-propelled interacting particle systems with roosting force, Math. Mod. Meth. Appl. Sci. 20(2010) 1533-1552.

[15] J.A. Carrillo, G. Toscani, Contractive probability metrics and asymptotic behavior of dissipative kinetic equations, Riv. Mat. Univ. Parma 6(2007) 75-198.

[16] Y.-L. Chuang, M.R. D’Orsogna, D. Marthaler, A.L. Bertozzi, L. Chayes, State transitions and the continuum limit for a 2D interacting, self-propelled particle system, Physica D 232(2007) 33-47. 
[17] Y.-L. Chuang, Y.R. Huang, M.R. D’Orsogna, A.L. Bertozzi, Multi-vehicle flocking : scalability of cooperative control algorithms using pairwise potentials, IEEE International Conference on Robotics and Automation (2007) 2292-2299.

[18] I. D. Couzin, J. Krause, R. James, G. D. Ruxton and N. R. Franks, Collective Memory and Spatial Sorting in Animal Groups, J. theor. Biol. 218(2002) 111.

[19] I.D. Couzin, J. Krause, N.R. Franks, S.A. Levin, Effective leadership and decision making in animal groups on the move, Nature 433(2005) 513-516.

[20] F. Cucker and S. Smale. Emergent behavior in flocks. IEEE Trans. Automat. Control $52(2007) 852-862$.

[21] A. Czirok, H.E. Stanley, T. Vicsek, Spontaneously ordered motion of self-propelled particles, J. Phys. A: Math. Gen. 30(1997) 1375-1385.

[22] P. Degond, A. Frouvelle, J.-G. Liu, Macroscopic limits and phase transition in a system of self-propelled particles, preprint arXiv:1109.2404v1.

[23] P. Degond, S. Motsch, Continuum limit of self-driven particles with orientation interaction, Math. Mod. Meth. Appl. Sci. 18(2008) 1193-1215.

[24] R. Dobrushin, Vlasov equations, Funct. Anal. Appl. 13(1979) 115-123.

[25] M.R. D’Orsogna, Y.-L. Chuang, A.L. Bertozzi, L. Chayes, Self-propelled particles with soft-core interactions : Patterns, stability and collapse, Phys. Rev. Lett. 96(2006) $104302-1 / 4$.

[26] W. Ebeling, U. Erdmann, Nonequilibrium statistical mechanics of swarms of driven particles, Complexity 8(2003) 23-30.

[27] A. Frouvelle, J.-G. Liu, Dynamics in a kinetic model of oriented particles with phase transition, Preprint arXiv:1101.2380v1.

[28] G. Grégoire, H. Chaté, On set of collective and cohesive motion, Phys. Rev. Lett. $92(2004) 025702-1 / 4$.

[29] S.-Y. Ha, J.-G. Liu, A simple proof of the Cucker-Smale flocking dynamics and meanfield limit, Comm. Math. Sci. 7(2009) 297-325.

[30] S.-Y. Ha, K. Lee, D. Levy, Emergence of Time-Asymptotic Flocking in a Stochastic Cucker-Smale System, Comm. Math. Sci. 7(2009) 453-469. 
[31] S.-Y. Ha, E. Tadmor, From particle to kinetic and hydrodynamic descriptions of flocking, Kinetic and Related Models 1(2008) 415-435.

[32] H. Hildenbrandt, C. Carere, C.K. Hemelrijk, Self-organised complex aerial displays of thousands of starlings: a model, Behav. Ecol. 21(2010) 1349-1359.

[33] A. Huth, C. Wissel, The Simulation of the Movement of Fish Schools, Journal of Theoretical Biology 156(1992) 365385.

[34] H. Levine, W.-J. Rappel, I. Cohen, Self-organization in systems of self-propelled particles, Phys. Rev. E 63(2000) 017101-1/4.

[35] S. Motsch, E. Tadmor, A new model for self-organized dynamics and its flocking behavior, J. Stat. Phys. 144(2011) 923-947.

[36] H. Neunzert, The Vlasov equation as a limit of Hamiltonian classical mechanical systems of interacting particles, Trans. Fluid Dynamics, 18(1977) 663-678.

[37] J. Parrish, L. Edelstein-Keshet, Complexity, pattern and evolutionary trade-offs in animal aggregation, Science 294(1999) 99-101.

[38] T. Vicsek, A. Czirok, E. Ben-Jacob, I. Cohen, O. Shochet, Novel type of phase transition in a system of self-driven particles, Phys. Rev. Lett. 75(1995) 1226-1229.

[39] C. Villani, Topics in optimal transportation, Graduate Studies in Mathematics, Amer. Math. Soc, Providence, 58 (2003). 